\title{
THE RECIPROCAL SPHERICAL ABERRATION OF AN OPTICAL SYSTEM INCLUDING HIGHER ORDERS
}

\author{
By Harold F. Bennett
}

The aberration present when an axial point is imaged by a centered system of spherical optical surfaces may be expressed by any one of a number of power series. In this paper the reciprocal of the distance between the intersection of a ray with the axis and a fixed point on the axis is expressed as a series in $h$, where $h$ is the perpendicular distance from the ray to the fixed point.

Formulas are derived by which the constant coefficients of the series expressing the aberration in the image space of a single spherical surface may be computed if the corresponding coefficients for the object space are known, the fixed point of reference being the center of curvature of the surface. These formulas can not be used in the case of a plane surface since there is no center of curvature. Accordingly, after developing transfer formulas by which the aberration may be referred to a new point of reference, a second set of formulas is derived in which the point of reference is taken as the vertex of the surface. A final set of formulas expresses the longitudinal in terms of the reciprocal aberration.

As a numerical example, the computation of the aberration of the third, fifth, and seventh orders of an ordinary photographic "landscape" lens is given in full. Its results compare favorably with those of a trigonometric tracing of rays in which seven place tables were used.

In conclusion the convergence of these series is briefly discussed and some relations to diffraction theory are pointed out.

\section{CONTENTS}

I. Introduction

1. Spherical aberration 188

2. Series expressions of longitudinal spherical aberration

3. Methods of evaluating the constants

4. Reciprocal spherical aberration

5. Historical sketch and bibliography 191

6. Notation and sign convention 192

II. The reciprocal aberration of a single spherical surface referred to the center of curvature.

1. Preliminary statement of problem

2. Derivation of the formulas

3. Discussion

(a) Inherent and propagated aberration

(b) Notes on numerical applications

(c) Roots, anomalous points, and specific examples...-

III. Transfer formulas

1. Derivation

2. Notes on generality

3. Transfer formulas involving powers of $n$

IV. The reciprocal aberration of a single surface referred to the vertex.-

1. Derivation of the formulas.

2. Discussion.

V. Inversion to longitudinal spherical 
VI. Numerical example

1. Details of construction of the lens

3. Transfer from center of curvature to center of curvature; first surface to second

4. Second surface and transfer to third; center of curvature to vertex

5. Third surface and inversion to longitudinal aberration.

6. Comparison with ray tracing

VII. Discussion

1. Convergence of aberration series

2. Relation to path difference equations.

3. Conclusion

\section{INTRODUCTION}

\section{SPHERICAL ABERRATION}

Rays of monochromatic light proceeding from a luminous point and passing through a lens are generally focussed not in a single image

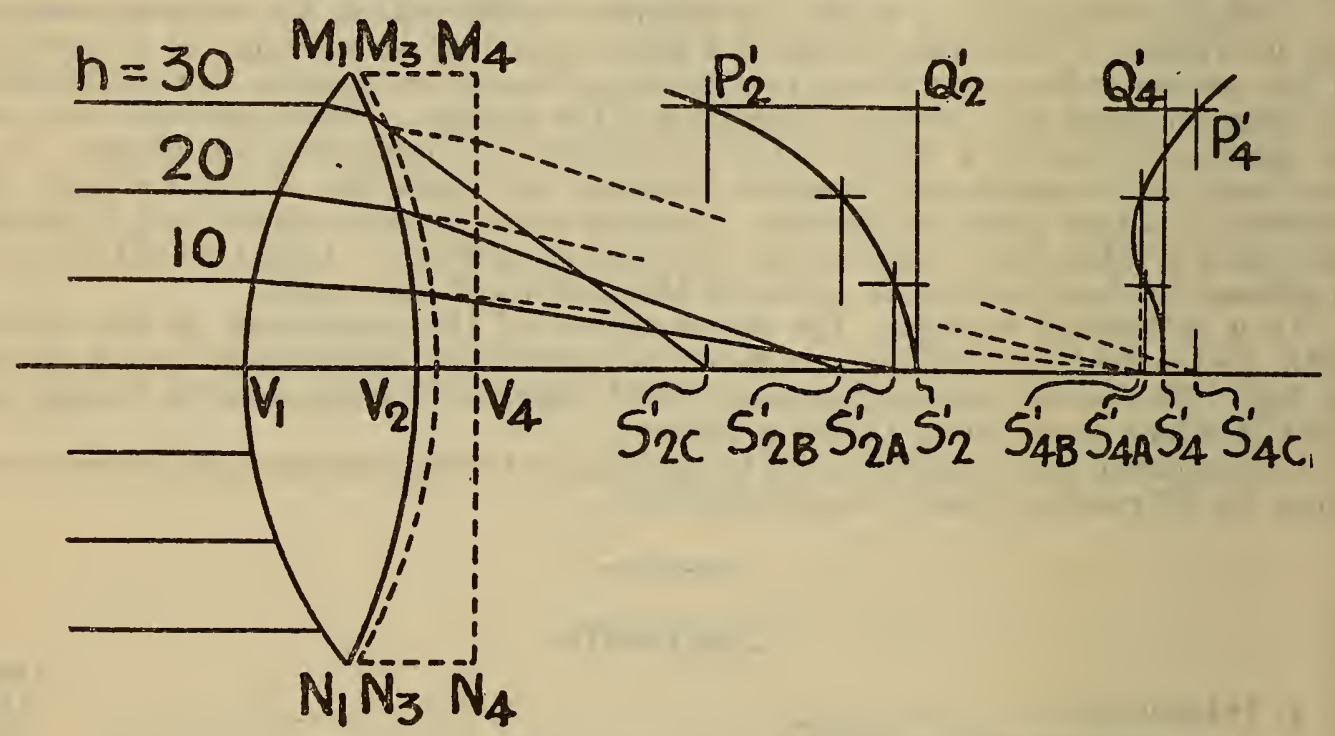

FIGURE 1.-Spherical aberration of a single lens and of a doublet

The paths of three rays when the concave lens is not present are shown in full lines. The concave lens and the paths of the rays if both lenses are present are shown in dotted lines. All parts of this flgure are drawn in proportion to values obtained by an actual calculation. ( $n_{1,2}=1.54$; $n_{3,4}=1.63$.)

point, but at varying distances from the lens according to the zone of the lens traversed. In the case of an object point on the axis of a centered system of spherical surfaces (the plane being considered a sphere of infinite radius) this defect in imagery is called "spherical aberration." Points off the axis are affected by the same aberration, but they are also affected by other aberrations, the presence of which makes analysis more difficult. The term "spherical aberration" is sometimes used more broadly to include these extra-axial aberrations and sometimes rather loosely in referring to the axial aberration where nonspherical surfaces are involved. In the present paper "spherical aberration" in the stricter sense of the word is discussed and a method is given for computing and expressing it quantitatively.

As an example of spherical aberration consider the portion of Figure 1 drawn in full lines. Light from an infinitely distant object point is 
incident upon the lens $M_{1} N_{1}$, and the three rays shown are refracted at the two surfaces so as to intersect the axis at ${S^{\prime}}_{2 A}, \mathrm{~S}_{2 B}^{\prime}$, and $\mathrm{S}_{2 C}$, which are the foci for these respective zones. If perpendiculars to the axis are drawn at these points, their intersections with lines parallel to the axis through the points of incidence on the first surface form the locus of the curve $S^{\prime}{ }_{2} P^{\prime}{ }_{2}$ which may be considered as representing both in direction and magnitude the spherical aberration in the image. For example, the distance $Q_{2}^{\prime} P_{2}{ }_{2}$ is the measure of the longitudinal spherical aberration of the ray at $h_{1}=30$. The dotted lines illustrate the method of correcting the spherical aberration by the insertion of a negative component, $M_{3}$. . $N_{4}$, of suitable design.

\section{SERIES EXPRESSIONS OF LONGITUDINAL SPHERICAL ABERRATION}

The aberration of a lens may be expressed quantitatively by giving the location of one or more points on the curve or by an equation for the curve. Such an equation is customarily written as an infinite series in which the terms beyond a certain order are neglected; thus the aberration represented by the curve $S_{4}^{\prime} P^{\prime}{ }_{4}$ (fig. 1 ) may be expressed as follows:

$$
\text { Long. spher. }=S^{\prime}-s^{\prime}=A^{\prime} h^{2}+B^{\prime} h^{4}+C^{\prime} h^{6}+\ldots
$$

where $S^{\prime}-s^{\prime}$ represents the abscissa and $h$ the ordinate, and where $s^{\prime}, A^{\prime}, B^{\prime}, C^{\prime}, \ldots$, are constants. The odd powers of $h$ are absent because of axial symmetry.

The number of terms of the series which it is necessary to retain depends upon the conditions of the problem in hand. The straight line, $S^{\prime}{ }_{4} Q^{\prime}{ }_{4}$, given by the equation $S^{\prime}=s^{\prime}$ is a first approximation which is exact only for infinitesimal apertures. A value of the constant $A^{\prime}$ may then be found such that the parabola $S^{\prime}=s^{\prime}+A^{\prime} h^{2}$ gives a sufficiently close approximation to the curve up to some aperture depending upon the accuracy required. Each of the terms of higher order has the property of remaining quite small for small apertures and then beginning to increase rapidly at some finite value of $h$. Accordingly, if the accuracy is not to diminish as larger and larger apertures are considered, the fifth order ${ }^{1}$ term and then the seventh order ${ }^{1}$ term must be taken into account, and so on.

In the present example $A^{\prime}$ is negative while $B^{\prime}$ and $C^{\prime}$ are positive, causing the upper portion of the curve to turn in the positive direction.

The same aberration, it is evident, could quite as legitimately be represented by one of a number of other curves and its corresponding

1 The longitudinal spherical aberration which varies as the $i$ th power of the aperture $(i=2,4,6 \ldots$. corresponds to an angular aberration (see equation (3), p. 194) which varies as the $(i+1)$ th power of the aperture and sponds to an angular aberration (see equation (3), p. 194) which varies as the $(i+1)$ th power of the aperture and
also to a path difference which varies as the $(i+2)$ th power of the aperture. The corresponding reciprocal spherical aberration (see Sec. I, 4) varies also as the $i$ th power of the aperture, but the lateral aberration varies as the $(i+1)$ th power. These differences of order of the expressions which refer in different manners - to the same aberration give rise to a difficulty in nomenclature. To avoid this the use of the terms primary, secondary, tertiary, etc., has been proposed. These designations not only become somewhat awkward for the higher-order aberrations treated in this paper, but some confusion might occur because primary and secondary already have special meanings in connection with astigmatism and curvature of field.

In the original papers of $\mathrm{L}$. Seidel the aberrations are measured by their lateral value so that the terms for spherical aberration assume the orders $3,5,7$, etc. In view of this and the general acceptance of Seidel's work it has been considered as desirable to designate a particular term of spherical aberration by the order which the corresponding lateral geometric aberration assumes. The expression " the third order longitudinal (or reciprocal) aberration" may then be interpreted as referring to "the longitudinal (or reciprocal) aberration which corresponds to the third order lateral aberration," and similarly for the terms of higher

order. thus corresponds exactly to the above designation of order $(i+1)$. 
equation, for instance by a curve the ordinates of which are equal to the heights of incidence on the last surface instead of the first. For any particular ray the total aberration would necessarily be the same according to either equation but, since the two values of $h$ are not directly proportional, different portions of the total aberration would be attributed to the different orders. It can be shown that the various orders differ, even if only a single surface is considered, according as the ordinate is defined as the height of incidence upon the surface, as the height of intersection with a plane tangent at the vertex, as the distance from the vertex or from the center of curvature of the surface measured along a perpendicular to the ray, as the arc, tangent, or sine of the angle of inclination of the ray with the axis, or as one of a number of other functions of the aperture. Upon investigation it is found that the third order coefficient, $A^{\prime}$, is identical in each group in which (as for the first three cases mentioned) the ordinate reduces to the same infinitesimal for very small apertures. For large apertures, however, the third order for a particular ray differs in the several equations insofar as $h^{2}$ is slightly different. Thus it may be seen that, if the expressions "third order," "fifth order," etc., are to have exact significance, the exact definition of the aperture must be indicated. Moreover, direct comparisons between different lenses can be made only when the aberrations of both are expressed in exactly the same manner.

\section{METHODS OF EVALUATING THE CONSTANTS}

It has been pointed out that successively closer approximations to the aberration curve can be obtained by evaluating additional terms of the series (equation (1)). The usual methods of evaluating these coefficients are of three general types. First, if the lens has actually been constructed, a number of narrow beams of light may be singled out, as in the Hartmann test, by a diaphragm with several small holes, and their positions after traversing the lens may be determined photographically or otherwise. Second, from the specifications of the lens system the theoretical position of several rays may be found by trigonometric ray tracing. In either case an empirical curve or equation is fitted to the discrete points thus obtained. Third, trigonometric relations, such as those used in ray tracing, are expanded as Taylor's or similar series in ascending powers of whatever function of the aperture is chosen as the parameter. The aberration coefficients are thereby expressed as furctions of the object distance and of the constants of the lens system. The present investigation is of this third type, which is usually called the algebraic or analytical method.

Ray tracing gives directly the total longitudinal aberration of one or more individual rays for any given lens. The algebraic method, however, is often more useful to the lens designer in that it reveals more fully the portion of the final aberration which is contributed by each surface of the lens and suggests in what manner the design should be altered. Moreover, very often when the design is altered only a small part of the computation need be repeated. The algebraic method has been considered objectionable because the third order equations are not sufficiently exact while if higher order terms are included the computation becomes too laborious. It is believed, however, that frequently the additional information is worth the labor 
involved, and, accordingly, formulas are given for enough orders so that sufficient accuracy may be attained in practically all cases.

\section{RECIPROCAL SPHERICAL ABERRATION}

The frequent occurrence of reciprocal distances in optical equations has suggested that the spherical aberration formulas might be simplified in form if the aberration instead of being expressed as a difference between the focal lengths of different zones were expressed as a difference between the powers of the reciprocals of the focal lengths.

An idea of the relation of the reciprocal aberration to the longitudinal may be gained as follows: Take for a moment the back image distance $V_{4}{S^{\prime}}_{4}=s^{\prime}{ }_{4}$ (fig. 1) as the unit of length. Plot a curve with the same ordinates as $S^{\prime}{ }_{4} P^{\prime}{ }_{4}$, but with abscissas equal to the reciprocals of those of this curve when measured from the vertex, $V_{4}$, as the origin. Where the abscissa of the old curve is slightly less than one that of the new is slightly more than one. Thus it is easily seen that the new curve will be very similar to $S^{\prime}{ }_{4} P^{\prime}{ }_{4}$ but reversed with with respect to $S^{\prime}{ }_{4} Q^{\prime}{ }_{4}$. Since the aberration is measured by the departure from the vertical straight line, the reciprocal aberration in any case is opposite in sign to the longitudinal aberration. The statements regarding equations expressing aberration, which were made in the two preceding sections ( 2 and 3 ), apply directly to the new curve except that other symbols, to be introduced later (see equations (2) and $\left(2^{\prime}\right)$ ) should replace the ones in equation (1). If a different unit of length is used the reciprocal aberration curve will merely be changed in scale in the horizontal direction. If, however, a different origin be selected, the curve will also be changed in shape.

\section{HISTORICAL SKETCH AND BIBLIOGRAPHY}

The theory of third-order aberrations is treated in the majority of books on geometrical optics. Among these Conrady ${ }^{2}$ is unexcelled from a practical standpoint, while Von Rohr ${ }^{3}$ gives a more elegant mathematical development and also includes an extensive bibliography.

Fifth-order terms for rays in the axial plane were published by Keller $^{4}$ and by Bauer. ${ }^{5}$ Their expressions are not strictly accurate, however, in that $h$ is not exactly defined. Kerber ${ }^{6}$ derived the fifth order reciprocal aberration of a single surface defining $h$ as the height of incidence. Schupmann ${ }^{7}$ used a fifth order term which he credited to Kerber. Von Rohr ${ }^{8}$ and König derived the fifth order term by Abbe's method of invariants with the angle of inclination of the ray as the variable. Risco ${ }^{9}$ extended these equations to apply to aspherical surfaces. Smart ${ }^{10}$ published terms of the fifth order, but unfortunately a number of his equations appear to be in error.

\footnotetext{
A. E. Conrady, Applied Optics and Optical Design, pt. 1, 518 pp., Oxford Univ. Press, 1929.

M. Von Robr, editor, Die Bilderzeugung in optischen Instrumenten, J. Springer, Berlin, 1904. Also translation by R. Kanthack, His Majesty's Stationery Office, London, 1920.

¿ G. A. Keller, Zur Dioptrik, Entwicklung der Glieder fünfter Ordnung. 24 pp., C. R. Schurich, Munich, 1865.

${ }_{5}^{5}$ K. L. Bauer, Carl's Reportorium f. Phys. Tech., vol. 1, pp. 219-241, 1886.

A. Kerber, Centr. Z. f. Opt. u. Mech., vol. 7, pp. 217-218, 1886.

7 L. Schupmann, Die Medial-Fernrohre. B. G. Teubner, Leipzig, 1899.

$8 \mathrm{M}$. Von Rohr, pp. 238-244. See footnote 3.

- M. Martinez Risco, Estudios Generales sobre Aberración Esferica de Orden Superior, Anales Soc. Espanola Fis. y Quim vol. 25 pt. 1, pp. 100-136, 1927.

10 E. H. Smart, Phil. Mag., vol. 20, pp. 82-91, 1910.
} 
Early investigators, notably Petzval, may have been in possession of formulas of higher orders than the fifth, ${ }^{11}$ but it is not known that any of these have been published. It is true, however, that investigations of aberrations from somewhat different standpoints have been extended to fifth and higher orders. Most of these are based upon the eikonal function, which depends upon the variation of path length through the lens.

\section{NOTATION AND SIGN CONVENTION}

A thoroughly consistent and complete system of notation for optical equations is, unfortunately, not available. In the present paper the aims have been to follow, in general, one of the systems already in use and to use as small a number of symbols as is compatible with clarity and brevity in presentation. In particular may be mentioned the use of the Greek characters $\beta$ and $\zeta$ with superscripts $\left(\beta^{(3)}, \beta^{(5)}\right.$, etc.) as constant coefficients of the various orders of reciprocal spherical aberration. (See equation (2) and footnote 1, p. 189.)

The original direction of the light is assumed to be from left to right. The refracting and reflecting surfaces are numbered in the order in which they are encountered by the light and are indicated by numerical subscripts. The well-known idea of regarding reflection as a special case of refraction in which the index ratio is -1 has been extended to include absolute indices (and indices relative to air), the index of any medium being considered as negative in sign if the light travels through it in a negative direction. ${ }^{12}$ Nevertheless, any other self-consistent sign convention may be used if preferred.

A list of the characters with their meanings is given herewith. Unprimed letters denote magnitudes in the object space, primed letters the corresponding magnitude in the image space. If the two are identical, as in the case of $r$ or $\phi$, the character is always written unprimed. In the following summary the primed character is omitted in most cases, it being assumed that its meaning is easily deduced from the definition of the corresponding unprimed character.

\section{ENGLISH LETTERS}

$A, B, C, \ldots .=$ the third order, fifth order, seventh order, . . . ., coefficients of longitudinal spherical aberration, positive if the aberration tends to make the rim ray cross the axis to the right of the paraxial focus.

$c=$ a subscript meaning "referred to the center of curvature" of the appropriate surface.

$d=$ the distance from the old point of reference to the new one to which the transfer is made, positive if to the right.

$h=$ (in Introduction) linear aperture, subject to a variety of definitions.

11 See, for instance, Trans. Opt. Soc. London, vol. 22, p. 214; 1920-21. Also Berek, Grundlagen der praktischen Optik, p. 43. Walter de Gruyter Co., Berlin, 1930.

${ }_{12}$ This convention is also applicable in path length equations as may be seen from the following simple example. Suppose a ray from some point $A$ is reflected back to $A$ from a mirror at $B$. The frst distance, $A$ to $B$ or $A B$, being positive, the distance $B$ to $A$ or $\mathrm{BA}$, after refection must be negative. Adding the distances together would give zero for the path length. However, if the index be considered negative after an odd number of refections and, as is universally done, the distance in each medium be multiplied by the index of refraction to get the equivalent path length in air the result is: Equiv. path length $=n A B+n^{\prime} \mathrm{BA}$

$$
\begin{aligned}
& =n \mathrm{AB}+n \mathrm{BA} \\
& =2 n \mathrm{BB}
\end{aligned}
$$

which is obviously correct.

An alternative sign convention in which some of the angles are considered as obtuse after reflection has been proposed by T. Smith in Trans. Opt. Soc. London, vol. 27, pp. 312-316, 1925-26. 
$h=$ the distance from the point of reference or origin of coordinates to a ray, measured along a perpendicular to the ray and positive if upward.

$i, j=$ generalized integers.

$n=$ the index of refraction of a medium, usually taken relacive to air, to be given a negative sign if che light traverses it from right to left.

$r=$ the radius of curvature of a speherical surface, positive if the surface is concave to the right.

$s=$ the distance from the vertex of a surface wo the paraxial object point for that surface, positive if to the right.

$t=s-r=$ the distance from the center of curvature of a surface to the paraxial object point for that surface, positive if to the right.

$S=$ the distance from the vertex of a surface to the intersection of a ray in the object pencil with the axis; reduces to $s$ as the aperture approaches zero.

$T=S-r=$ the distance from the center of curvature of a surface to the intersection of a ray in the object pencil with the axis; reduces to $t$ as the aperture approaches zero.

$v=a$ subscript meaning "referred to the vertex" of the appropriate surface.

\section{GREEK LETTERS}

$\alpha=$ the acute angle between a ray and the axis, positive if the ray lies above the axis to the left of the point of intersection.

$\beta(i)=$ the coefficient of reciprocal spherical aberration of the $i$ th order, in the series referred to the center of curvature, usually of the sign opposite that of the corresponding longitudinal aberration coefficient.

$\zeta^{(i)}=$ the coefficient of reciprocal spherical aberration of the $i$ th order in the series referred to the vertex of the lens surface, usually of the sign opposite that of the corresponding longitudinal aberration coefficient.

$\Theta=$ ithe reciprocal of $T(q . v$.$) .$

$\vartheta, \vartheta^{\prime}=$ the angle of incidence, the angle of emergence; that is, the acute angle between a ray and the normal to the surface at the point of incidence, positive if the ray lies above the normal to the right of the surface.

$\kappa=$ the constant of transfer to a new point of reference.

$=$ the ratio of the new paraxial reciprocal object (or image) distance to the old paraxial reciprocal image distance.

$\kappa, \kappa^{\prime}$ in Part IV take on the special meaning that the transfer is made from the vertex to the center of curvature of a single surface, $\kappa$ applying to the object pencil and $\kappa^{\prime}$ to the image pencil.

$\mu=$ relative index $=n / n^{\prime}$.

$\mu^{\prime}=$ relative index $=n^{\prime} / n$.

$\rho, \sigma, \tau=$ the reciprocals of $r, s$, and $t$.

$\Sigma=$ the reciprocal of $S$.

$\phi=$ the central angle or the angle between the radius drawn to the point of incidence and the axis, positive if the radius lies above the axis to the left of the center of curvature.

$\Psi=$ reciprocal object distance from any point of reference on the axis.

\section{THE RECIPROCAL ABERRATION OF A SINGLE SPHER- ICAL SURFACE REFERRED TO THE CENTER OF CURVA- TURE}

\section{PRELIMINARY STATEMENT OF PROBLEM}

A set of formulas will first be derived which express the reciprocal aberration coefficients of the refracted or image pencil of any spherical surface when the corresponding constants of the incident or object pencil for that surface are known, the point of reference being chosen at the center of curvature of the surface. 
In Figure 2, VS represents the axis of symmetry of a lens system of which one surface, with vertex at $V$ and center of curvature at $C$, is shown in cross section. In a symmetrical pencil of rays incident upon this surface, consider the ray $Q P$, incident at the point $P$. Let this ray (extended if necessary) intersect the axis at the point $S$, removed from the center of curvature by a distance $T$, positive if to the right. Let $h_{c}$ denote the perpendicular distance from the center of curvature to the ray, positive if upward, and $r$ the radius of curvature of the surface, positive if the surface is concave toward the right.

Let $\beta^{(3)}, \beta^{(5)}, \beta^{(7)}$, etc., be constants, such that if $h_{c}$ is given, $T$ is determined to as close an approximation as may be desired by retaining enough terms of the series:

$$
\frac{1}{T}=\frac{1}{t}+\beta^{(3)} h_{c}{ }^{2}+\beta^{(5)} h_{c}^{4}+\beta^{(7)} h_{c}^{6}+\ldots
$$

Here $t$ is the limiting value of $T$ as $h_{c}$ approaches zero; that is, the paraxial value of $T, \beta^{(3)} h_{c}{ }^{2}$ is the third order term of reciprocal aberra-

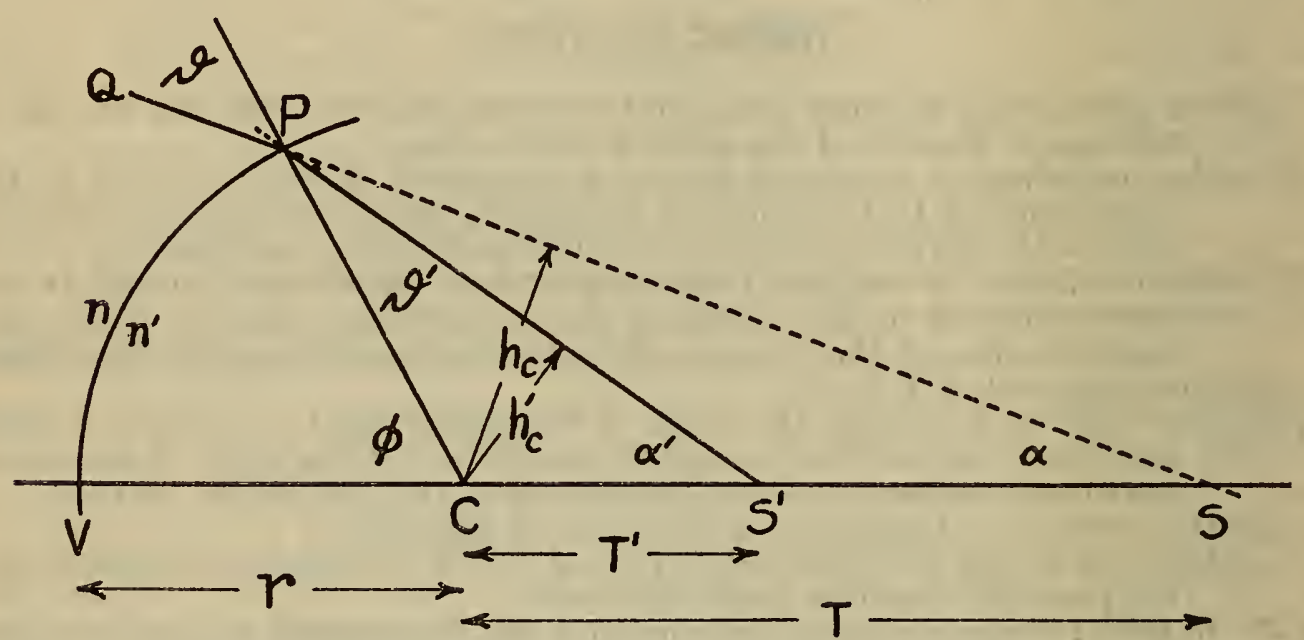

FIGURE 2.-Refraction of a ray at a single surface

tion, $\beta^{(5)} h_{c}^{4}$ is the fifth order, etc. For convenience in writing the equation, let $\frac{1}{T}=\theta$ and $\frac{1}{t}=\tau$, then

$$
\Theta=\tau+\beta^{(3)} h_{c}{ }^{2}+\beta^{(8)} h_{c}{ }^{4}+\beta^{(7)} h_{c}^{6}+\beta^{(8)} h_{c}^{8}+\ldots
$$

The angles $\alpha, \vartheta$, and $\phi$ are shown in Figure 2. They are defined by the following equations, apparent from the geometry of the figure

$$
\begin{aligned}
\sin \alpha & =\frac{h_{c}}{T}=\theta h_{c} \\
& =\tau h_{c}+\beta^{(3)} h_{c}{ }^{3}+\beta^{(5)} h_{c}{ }^{5}+\ldots \\
\sin \vartheta & =\frac{h_{c}}{r}=\rho h_{c} \\
\phi & =\alpha+\vartheta
\end{aligned}
$$

where $\rho$ is written for $\frac{1}{r}$. 
Let the ray pass from a medium of index $n$ into one of index $n^{\prime}$ and write for convenience $\frac{n^{\prime}}{n}=\mu^{\prime} .{ }^{13}$ Denote the various functions of the refracted ray, $P S^{\prime}$, by priming those symbols which when unprimed represent the corresponding functions of the incident ray. Then:

$$
\begin{gathered}
\theta^{\prime}=\tau^{\prime}+\beta^{\prime(3)} h_{c}^{\prime}{ }_{c}^{2}+\beta^{\prime(5)} h_{c}^{\prime}{ }_{c}^{4}+\ldots \\
\sin \alpha^{\prime}=\tau^{\prime} h^{\prime}{ }_{c}+\beta^{\prime(3)} h^{\prime}{ }_{c}^{3}+\beta^{\prime(5)} h_{c}^{\prime}{ }_{c}^{5}+\ldots \\
\sin \vartheta^{\prime}=\rho h^{\prime}{ }_{c}
\end{gathered}
$$

and

$$
\alpha^{\prime}+\vartheta^{\prime}=\phi=\alpha+\vartheta
$$

\section{DERIVATION OF THE FORMULAS}

By Snell's law and equations (4) and (4')

$$
\sin \vartheta=\rho h_{c}=\mu^{\prime} \rho h^{\prime}{ }_{c}
$$

From this $h_{c}=\mu^{\prime} h^{\prime}{ }_{c}$; then substituting in equation (3):

$$
\sin \alpha=\mu^{\prime} \tau h^{\prime}{ }_{c}+\mu^{\prime 3} \beta^{(3)} h^{\prime}{ }_{c}^{3}+\mu_{\mu^{\prime 11}} \beta^{(11)} \beta^{(5)} h_{c}^{\prime}{ }_{c}{ }^{5}+\mu^{\prime 1}{ }^{(7)} h^{\prime}{ }_{c}{ }^{7}+\mu^{\prime 9} \beta^{(9)} h^{\prime}{ }_{c}^{9}+
$$

The sines of $\alpha, \vartheta$, and $\vartheta^{\prime}$ have now been expressed as functions of the same variable, $h_{c}^{\prime}$. Of the possible methods of combining these to give sin $\alpha^{\prime}$, the unknown angle of equation (5), the following one using trigonometrical addition formulas and series expansions has been thought the most convenient $i_{i}^{14}$

by equation (6)

$$
\cos \vartheta=1-\frac{1}{2} \sin ^{2} \vartheta-\frac{1}{8} \sin ^{4} \vartheta-\ldots
$$

$$
\begin{aligned}
= & 1-\frac{1}{2} \mu^{\prime 2} \rho^{2} h_{c}^{\prime}{ }_{c}{ }^{2}-\frac{1}{8} \mu^{\prime 4} \rho^{4} h_{c}^{\prime}{ }_{c}^{4}-\frac{1}{16} \mu^{16} \rho^{6} h_{c}^{\prime}{ }_{c}^{8} \\
& -\frac{5}{128} \mu^{\prime 8} \rho^{8} h_{c}^{\prime}{ }_{c}^{8}-\frac{7}{256} \mu^{\prime 10} \rho^{10} h_{c}^{\prime}{ }_{c}^{10} \\
\cos \vartheta^{\prime}= & 1-\frac{1}{2} \rho^{2} h^{\prime}{ }_{c}^{2}-\frac{1}{8} \rho^{4} h^{\prime}{ }_{c}^{4}-\frac{1}{16} \rho^{6} h^{\prime}{ }_{c}^{6}-\ldots
\end{aligned}
$$

$\sin \left(\vartheta-\vartheta^{\prime}\right)=\sin \vartheta \cos \vartheta^{\prime}-\cos \vartheta \sin \vartheta^{\prime}$

$$
\begin{aligned}
= & \left(\mu^{\prime}-1\right) \rho h^{\prime}{ }_{c}-\frac{1}{2}\left(\mu^{\prime}-\mu^{2}\right) \rho^{3} h^{\prime}{ }_{c}^{3} \\
& -\frac{1}{8}\left(\mu^{\prime}-\mu^{\prime 4}\right) \rho^{5} h^{\prime}{ }_{c}^{5}-\frac{1}{16}\left(\mu^{\prime}-\mu^{\prime 6}\right) \rho^{7} h^{\prime}{ }_{c}^{7} \\
& -\frac{5}{128}\left(\mu^{\prime}-\mu^{\prime 8}\right) \rho^{9} h^{\prime}{ }_{c}^{9}-\frac{7}{256}\left(\mu^{\prime}-\mu^{\prime 10}\right) \rho^{11} h_{c}^{\prime}{ }_{c}^{11}
\end{aligned}
$$

13 The sense of the index ratio and the direction of the ray are thus mutually defined. If the ray passes through the refracting surface from left to right, then the index $n$ pertains to the medium on the left and vice versa. The sign convention for distances and angles is independent of the direction of propagation of light along the ray. The index is considered as negative if the light travels from right to leit. (See footnote 12 , p. 192.)

note 12 , p. 192.) that the higher orders may be ignored entirely. Moreover, it will doubtless be found that the method of derivation of the formulas is more easily followed if only the first one or two terms of each equation are taken into consideration. 
TABLE 1. $-\operatorname{Cos} \vartheta \cos \vartheta^{\prime}$

\begin{tabular}{|c|c|c|c|c|c|}
\hline 1 & $h^{\prime} c^{2}$ & $h^{\prime} c^{4}$ & $h^{\prime} c^{8}$ & $h^{\prime} c^{8}$ & $h^{\prime} c^{10}$ \\
\hline 1 & $-\frac{1}{2} \mu^{\prime 2} \rho^{2}$ & $-\frac{1}{8} \mu^{\prime \prime} \rho^{4}$ & $-\frac{1}{16} \mu^{\prime 5} \rho^{6}$ & $-\frac{5}{128} \mu^{\prime 8} \rho^{8}$ & $-\frac{7}{256} \mu^{\prime 10} \rho^{10}$ \\
& $-\frac{1}{2} \rho^{2}$ & $+\frac{1}{4} \mu^{\prime 2} \rho^{4}$ & $+\frac{1}{16} \mu^{\prime 4} \rho^{6}$ & $+\frac{1}{32} \mu^{\prime} \rho^{8}$ & $+\frac{5}{256} \mu^{\prime 8} \rho^{10}$ \\
& $-\frac{1}{8} \rho^{4}$ & $+\frac{1}{16} \mu^{\prime 2} \rho^{6}$ & $+\frac{1}{64} \mu^{\prime 4} \rho^{8}$ & $+\frac{1}{128} \mu^{\prime 6} \rho^{10}$ \\
& & $-\frac{1}{16} \rho^{8}$ & $+\frac{1}{32} \mu^{\prime 2} \rho^{8}$ & $+\frac{1}{128} \mu^{\prime 4} \rho^{10}$ \\
& & & $-\frac{5}{128} \rho^{8}$ & $+\frac{5}{256} \mu^{\prime 2} \rho^{10}$ \\
& & & & $-\frac{7}{256} \rho^{10}$ \\
\hline
\end{tabular}

$\cos \left(\vartheta-\vartheta^{\prime}\right)=\cos \vartheta \cos \vartheta^{\prime}+\sin \vartheta \sin \vartheta^{\prime}$

$$
=\text { the expansion }{ }^{15} \text { in Table } 1+\mu^{\prime} \rho^{2} h_{c}^{\prime 2}
$$

$$
\begin{aligned}
= & 1-\frac{1}{2}\left(\mu^{\prime}-1\right)^{2} \rho^{2} h^{\prime}{ }_{c}^{2}-\frac{1}{8}\left(\mu^{\prime 2}-1\right)^{2} \rho^{4} h_{c}^{\prime}{ }_{c}^{4} \\
& -\frac{1}{16}\left(\mu^{\prime 2}-1\right)^{2}\left(\mu^{\prime 2}+1\right) \rho^{6} h^{\prime}{ }_{c}^{6} \\
& -\frac{1}{128}\left(\mu^{\prime 2}-1\right)^{2}\left(5 \mu^{\prime 4}+6 \mu^{\prime 2}+5\right) \rho^{8} h_{c}^{\prime}{ }_{c} \\
& -\frac{1}{256}\left(\mu^{\prime 2}-1\right)^{2}\left(7 \mu^{\prime 6}+9 \mu^{\prime 4}+9 \mu^{\prime 2}+7\right) \rho^{10} h^{\prime}{ }_{c}^{10} \\
\cos \alpha= & 1-\frac{1}{2} \sin ^{2} \alpha-\frac{1}{8} \sin ^{4} \alpha-. . \\
= & \text { the expansion }{ }^{15} \text { in Table } 2 .
\end{aligned}
$$

\begin{tabular}{|c|c|c|c|c|c|}
\hline 1 & $h^{\prime}{ }_{c}^{2}$ & $h^{\prime} c^{4}$ & $h^{\prime} c^{6}$ & $h^{\prime} c^{8}$ & $h^{\prime} c^{10}$ \\
\hline 1 & $-\frac{1}{2} \mu^{\prime 2} \tau^{2}$ & $\begin{array}{l}-\mu^{\prime 4} \tau \beta^{(3)} \\
-\frac{1}{8} \mu^{\prime 4} \tau^{4}\end{array}$ & $\begin{array}{l}-\mu^{\prime 6} \tau \beta^{(6)}-\frac{1}{2} \mu^{\prime} 6 \beta^{(3) 2} \\
-\frac{1}{2} \mu^{\prime 6} \tau^{3} \beta^{(3)} \\
-\frac{1}{16} \mu^{\prime 6} \tau^{6}\end{array}$ & $\begin{array}{l}-\mu^{\prime} \tau \beta^{(i)}-\mu^{\prime 8} \beta^{(3)} \beta^{(5)} \\
-\frac{1}{2} \mu^{\prime} \tau^{3} \beta^{(5)}-\frac{3}{4} \mu^{\prime 8} \tau^{2} \\
\beta^{(3) \cdot 2} \\
-\frac{3}{8} \mu^{\prime 8} \tau^{5} \beta^{(3)} \\
-\frac{5}{128^{(3)}} \mu^{\prime} \tau^{8}\end{array}$ & $\begin{array}{l}-\mu^{\prime 10} \tau^{(8)}-\mu^{\prime 10} \beta^{(3)} \beta^{(7)} \\
\quad-\frac{1}{2} \mu^{\prime 10} \beta^{(5) 2} \\
-\frac{1}{2} \mu^{\prime 10} \tau^{3} \beta^{(7)}-\frac{3}{2} \mu^{\prime 10} \tau^{2} \beta^{(3)} \beta^{(3)}- \\
\frac{1}{2} \mu^{\prime 10} \tau \beta^{(3) 3} \\
-\frac{3}{8} \mu^{\prime 10} \tau^{5} \beta^{(b)}-\frac{15}{16} \mu^{\prime 10} \tau^{4} \beta^{(3) 2} \\
-\frac{5}{16} \mu^{\prime 10} \tau^{7} \beta^{(3)} \\
-\frac{7}{256} \mu^{\prime 10} \tau^{10}\end{array}$ \\
\hline
\end{tabular}

TABLE 2.-Expansion of $\cos \alpha$

[The coefficient of each power of $h^{\prime}{ }_{c}$ is the sum of all the quantities below it in the same column]

$$
\begin{aligned}
\sin \alpha^{\prime} & =\sin \left[\alpha+\left(\vartheta-\vartheta^{\prime}\right)\right] \\
& =\sin \alpha \cos \left(\vartheta-\vartheta^{\prime}\right)+\cos \alpha \sin \left(\vartheta-\vartheta^{\prime}\right) \\
& =\text { the expansion }{ }^{15} \text { in Table } 3 \\
& =\tau^{\prime} h^{\prime}{ }_{c}+\beta^{\prime(3)} h^{\prime}{ }_{c}^{3}+\beta^{\prime(5)} h^{\prime}{ }_{c}^{5}+\beta^{\prime(7)} h^{\prime}{ }_{c}^{7}+\ldots
\end{aligned}
$$

the last term being obtained from equation $\left(3^{\prime}\right)$.

15 This member of the equation has been written in tabular form for clearness and convenience. In Tables land 2 the quantities in a single column are to be considered as inclosed in brackets and multiplied by the power of $h$ at the top. In Tables 3 and 4 the quantity in each small rectangle in the body of the table is to be multiplied by the power of $h$ at the top of the column and also by the $\beta$-coefficient at the left end of the row. The quantity represented by the whole table is the sum of all these products. The products may be grouped either by row or by column (in this case the latter is more convenient) and the common factors taken out. 


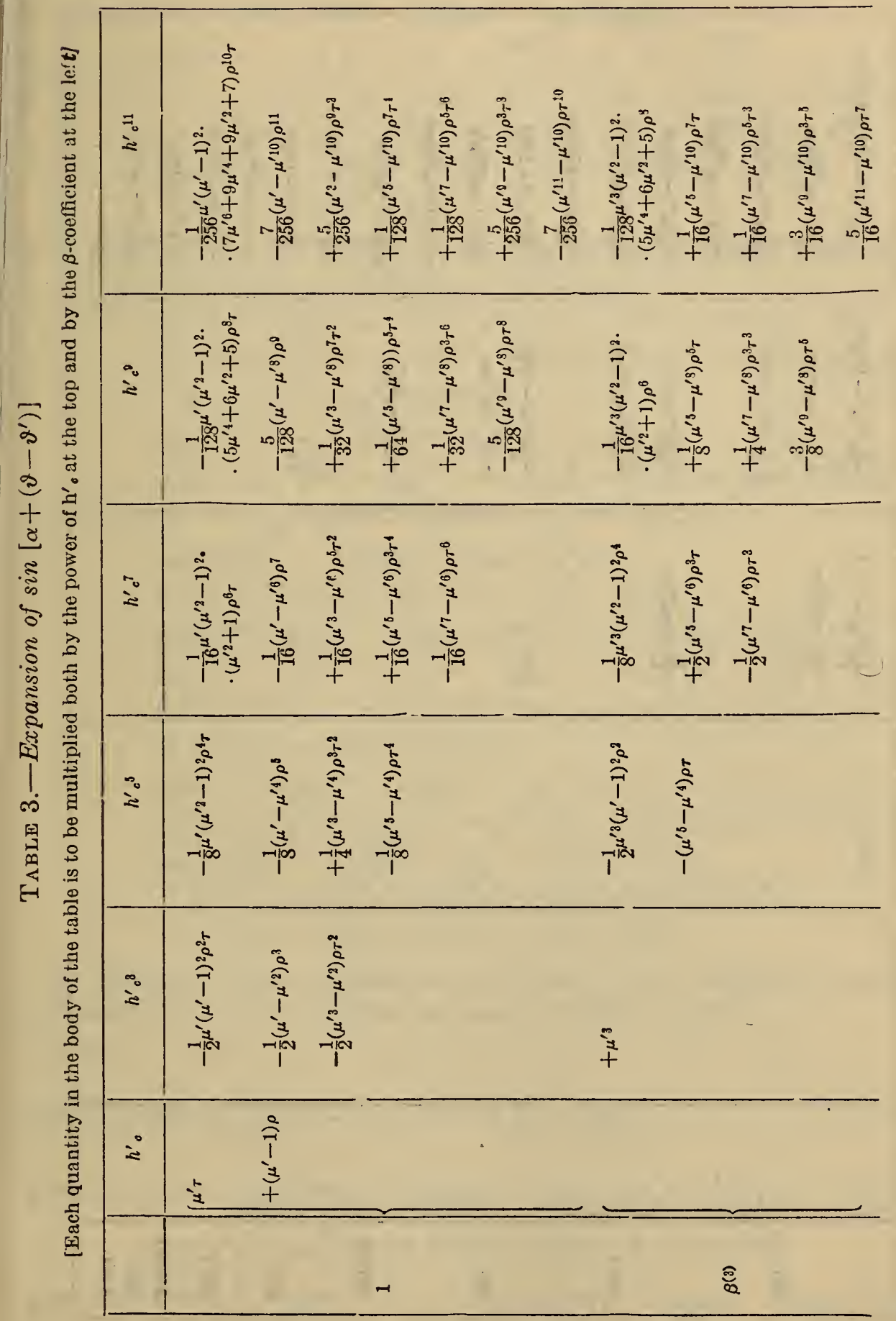




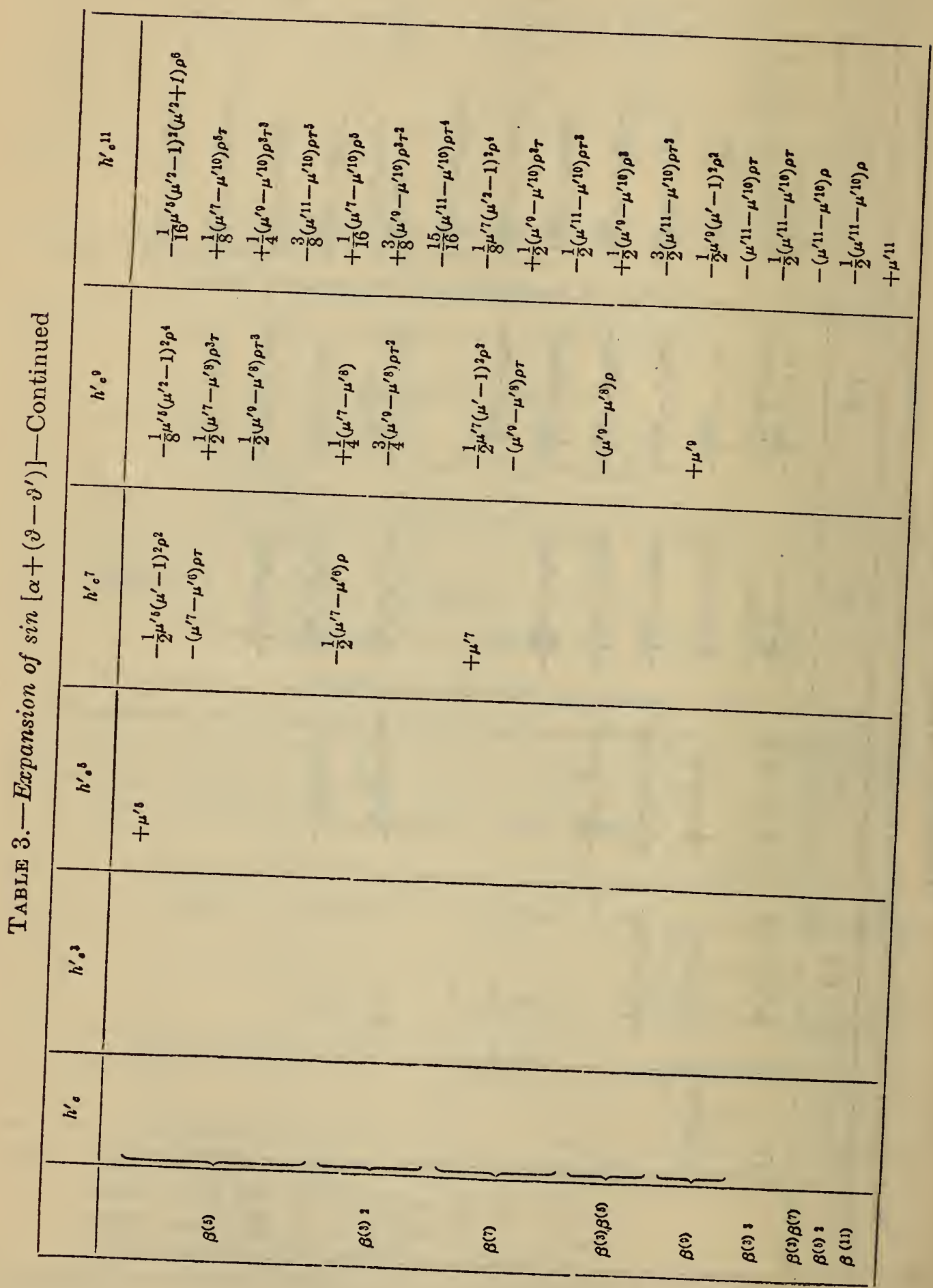


Since, to the order of approximation attained, this is true for any value of $h^{\prime}{ }_{c}$, the coefficients of like powers of $h^{\prime}{ }_{c}$ may be equated and are readily reduced to the following form:

$$
\begin{aligned}
& \tau^{\prime}=\mu^{\prime} \tau+\left(\mu^{\prime}-1\right) \rho \\
& \beta^{\prime(3)}=\mu^{\prime 3} \beta^{(3)}-1 / 2 \mu^{\prime} \rho\left[\left(\mu^{\prime}-1\right)^{2} \rho \tau+\left(\rho^{2}+\mu^{\prime 2} \tau^{2}\right)-\mu^{\prime}\left(\rho^{2}+\tau^{2}\right)\right] \\
& \beta^{\prime(5)}=\mu^{\prime 5} \beta^{(5)}-1 / 2 \mu^{\prime 3} \beta^{(3)}\left(\mu^{\prime}-1\right) \rho\left[\left(\mu^{\prime}-1\right) \rho+2 \mu^{\prime} \tau\right] \\
& -3 / 8 \mu^{\prime} \rho\left[\left(\mu^{\prime 2}-1\right)^{2} \rho^{3} \tau+\left(\rho^{2}-\mu^{\prime 2} \tau^{2}\right)^{2}-\mu^{\prime 3}\left(\rho^{2}-\tau^{2}\right)^{2}\right] \\
& \beta^{\prime(7)}=\mu^{77} \beta^{(7)}-1 / 2 \mu^{\prime 6} \beta^{(3) 2}\left(\mu^{\prime}-1\right) \rho-1 / 2 \mu^{\prime 5} \beta^{(5)}\left(\mu^{\prime}-1\right) \rho\left[\left(\mu^{\prime}-1\right) \rho+2 \mu^{\prime} \tau\right] \\
& -1 / 2 \mu^{\prime 3} \beta^{(3)} \rho\left[1 / 4\left(\mu^{\prime 2}-1\right)^{2} \rho^{3}-\mu^{\prime 2} \tau\left(\rho^{2}-\mu^{\prime 2} \tau^{2}\right)+\mu^{\prime 3} \tau\left(\rho^{2}-\tau^{2}\right)\right] \\
& -1 / 16 \mu^{\prime} \rho\left[\left(\mu^{\prime 2}-1\right)^{2}\left(\mu^{\prime 2}+1\right) \rho^{5} \tau+\left(\rho^{2}-\mu^{\prime 2} \tau^{2}\right)^{2}\left(\rho^{2}+\mu^{\prime 2} \tau^{2}\right)\right. \\
& \left.-\mu^{\prime 5}\left(\rho^{2}-\tau^{2}\right)^{2}\left(\rho^{2}+\tau^{2}\right)\right] \\
& \beta^{\prime(9)}=\mu^{\prime 9} \beta^{(9)}-\mu^{\prime 8} \beta^{(3)} \beta^{(5)}\left(\mu^{\prime}-1\right) \rho-1 / 2 \mu^{\prime 7} \beta^{(7)}\left(\mu^{\prime}-1\right) \rho\left[\left(\mu^{\prime}-1\right) \rho+2 \mu^{\prime} \tau\right] \\
& +\gamma_{4}^{\prime} \mu^{\prime 7} \beta^{(3) 2} \rho\left[\left(\rho^{2}-3 \mu^{\prime 2} \tau^{2}\right)-\mu^{\prime}\left(\rho^{2}-3 \tau^{2}\right)\right] \\
& \left.-1_{2} \mu^{\prime 5} \beta^{(5)} \rho\left[1 / 1 / 4 \mu^{\prime 2}-1\right)^{2} \rho^{3}-\mu^{\prime 2} \tau\left(\rho^{2}-\mu^{\prime 2} \tau^{2}\right)+\mu^{\prime 3} \tau\left(\rho^{2}-\tau^{2}\right)\right] \\
& -1 / 8 \mu^{\prime 3} \beta^{(3)} \rho\left[1 / 2\left(\mu^{\prime 2}-1\right)^{2}\left(\mu^{\prime 2}+1\right) \rho^{5}-\mu^{\prime 2} \tau\left(\rho^{2}-\mu^{\prime 2} \tau^{2}\right)\left(\rho^{2}+3 \mu^{\prime 2} \tau^{2}\right)\right. \\
& \left.+\mu^{\prime 5} \tau\left(\rho^{2}-\tau^{2}\right)\left(\rho^{2}+3 \tau^{2}\right)\right] \\
& -3_{128} \mu^{\prime} \rho\left[\left(\mu^{\prime 2}-1\right)^{2}\left(5 \mu^{\prime 4}+6 \mu^{2}+5\right) \rho^{7} \tau+\left(\rho^{2}-\mu^{\prime 2} \tau^{2}\right)^{2}\right. \text {. } \\
& \left.\cdot\left(5 \rho^{4}+6 \mu^{\prime 2} \rho^{2} \tau^{2}+5 \mu^{\prime 4} \tau^{4}\right)-\mu^{\prime 7}\left(\rho^{2}-\tau^{2}\right)\left(5 \rho^{4}+6 \rho^{2} \tau^{2}+5 \tau^{4}\right)\right] \\
& \beta^{\prime(11)}=\mu^{\prime 11} \beta^{(11)}-1 / 2 \mu^{\prime 10}\left[\beta^{(5) 2}+2 \beta^{(3)} \beta^{(7)}+\tau \beta^{(3) 3}\right]\left(\mu^{\prime}-1\right) \rho \\
& -1 / 2 \mu^{\prime 9} \beta^{(9)}\left(\mu^{\prime}-1\right) \rho\left[\left(\mu^{\prime}-1\right) \rho+2 \mu^{\prime} \tau\right] \\
& +1 / 2 \mu^{\prime 9} \beta^{(3)} \beta^{(5)} \rho\left[\left(\rho^{2}-3 \mu^{\prime 2} \tau^{2}\right)-\mu^{\prime}\left(\rho^{2}-3 \tau^{2}\right)\right] \\
& -1 / 2 \mu^{\prime 7} \beta^{(7)} \rho\left[1_{4}\left(\mu^{\prime 2}-1\right)^{2} \rho^{3}-\mu^{\prime 2} \tau\left(\rho^{2}-\mu^{\prime 2} \tau^{2}\right)+\mu^{\prime 3} \tau\left(\rho^{2}-\tau^{2}\right)\right] \\
& -1 / 16 \mu^{\prime 7} \beta^{(3) 2} \rho\left[\left(\rho^{4}+6 \mu^{\prime 2} \rho^{2} \tau^{2}-15 \mu^{\prime 4} \tau^{4}\right)-\mu^{\prime 3}\left(\rho^{4}+6 \rho^{2} \tau^{2}-15 \tau^{4}\right)\right] \\
& -1 / 8 \mu^{\prime 5} \beta^{(5)} \rho\left[1 / 2\left(\mu^{\prime 2}-1\right)^{2}\left(\mu^{\prime 2}+1\right) \rho^{5}-\mu^{\prime 2} \tau\left(\rho^{2}-\mu^{\prime 2} \tau^{2}\right)\left(\rho^{2}+3 \mu^{\prime 2} \tau^{2}\right)\right. \\
& \left.+\mu^{\prime 5} \tau\left(\rho^{2}-\tau^{2}\right)\left(\rho^{2}+3 \tau^{2}\right)\right] \\
& -1 / 16 \mu^{\prime 3} \beta^{(3)} \rho\left[1 / 8\left(\mu^{\prime 2}-1\right)\left(5 \mu^{\prime 4}+6 \mu^{\prime 2}+5\right) \rho^{7}-\mu^{\prime 2} \tau\left(\rho^{2}-\mu^{\prime 2} \tau^{2}\right)\right. \text {. } \\
& \text { - } \left.\left(\rho^{4}+2 \mu^{\prime 2} \rho^{2} \tau^{2}+5 \mu^{\prime 4} \tau^{4}\right)+\mu^{\prime 7} \tau\left(\rho^{2}-\tau^{2}\right)\left(\rho^{4}+2 \rho^{2} \tau^{2}+5 \tau^{4}\right)\right] \\
& -1 / \rho_{26} \mu^{\prime} \rho\left[\left(\mu^{\prime 2}-1\right)^{2}\left(7 \mu^{\prime 6}+9 \mu^{\prime 4}+9 \mu^{\prime 2}+7\right) \rho^{9} \tau\right. \\
& +\left(\rho^{2}-\mu^{\prime 2} \tau^{2}\right)^{2}\left(7 \rho^{6}+9 \mu^{\prime 2} \rho^{4} \tau^{2}+9 \mu^{\prime 4} \rho^{2} \tau^{4}+7 \mu^{\prime 6} \tau^{6}\right) \\
& \left.-\mu^{\prime 9}\left(\rho^{2}-\tau^{2}\right)^{2}\left(7 \rho^{6}+9 \rho^{4} \tau^{2}+9 \rho^{2} \tau^{4}+7 \tau^{6}\right)\right]
\end{aligned}
$$

If, instead, the quantities in Table 3 be factored the following equations (written only to the ninth order) result:

$$
\begin{aligned}
\tau^{\prime}= & \mu^{\prime}(\tau+\rho)-\rho \text { or }\left(\tau^{\prime}+\rho\right)=\mu^{\prime}(\tau+\rho) \\
\beta^{\prime(3)}= & \mu^{\prime 3} \beta^{(3)}+\frac{1}{2} \mu^{\prime}\left(\mu^{\prime}-1\right) \rho(\rho+\tau)\left(\rho-\mu^{\prime} \tau\right) \\
\beta^{\prime(3)}= & \mu^{\prime 5} \beta^{(5)}-\frac{1}{2} \mu^{\prime 3} \beta^{(3)}\left(\mu^{\prime}-1\right) \rho\left[\left(\mu^{\prime}-1\right) \rho+2 \mu^{\prime} \tau\right]+\frac{1}{8} \mu^{\prime}\left(\mu^{\prime}-1\right) \rho(\rho+\tau) \\
& \quad\left(\rho-\mu^{\prime} \tau\right)\left[\left(\mu^{\prime 2}+\mu^{\prime}+1\right) \rho^{2}-\mu^{\prime}(\mu-1) \rho \tau+\mu^{\prime 2} \tau^{2}\right] \\
\beta^{\prime(7)}= & \mu^{\prime 7} \beta^{(7)}-\frac{1}{2} \mu^{\prime 8} \beta^{(3) 2}\left(\mu^{\prime}-1\right) \rho-\frac{1}{2} \mu^{\prime 5} \beta^{(5)}\left(\mu^{\prime}-1\right) \rho\left[\left(\mu^{\prime}-1\right) \rho+2 \mu^{\prime} \tau\right] \\
& -\frac{1}{8} \mu^{\prime 3} \beta^{(3)}\left(\mu^{\prime}-1\right) \rho\left[\left(\mu^{\prime}+1\right)\left(\mu^{\prime 2} \quad 1\right) \rho^{3}+4 \mu^{\prime 2} \tau\left(\rho^{2}+\mu^{\prime} \tau^{2}\right)\right] \\
& +\frac{1}{16} \mu^{\prime}\left(\mu^{\prime}-1\right) \rho(\rho+\tau)\left(\rho-\mu^{\prime} \tau\right)\left[\left(\mu^{\prime 4}+\mu^{\prime 3}+\mu^{\prime 2}+\mu^{\prime}+1\right) \rho^{4}\right. \\
& \left.-\mu^{\prime}\left(\mu^{\prime 3}-1\right) \rho^{3} \tau+\mu^{\prime 2}\left(\mu^{\prime 2}+1\right) \rho^{2} \tau^{2}-\mu^{\prime 3}\left(\mu^{\prime}-1\right) \rho \tau^{3}+\mu^{\prime 4} \tau^{4}\right]
\end{aligned}
$$




$$
\begin{aligned}
\beta^{\prime(9)}= & \mu^{\prime 9} \beta^{(9)}-\mu^{\prime 8} \beta^{(3)} \beta^{(5)}\left(\mu^{\prime}-1\right) \rho-\frac{1}{2} \mu^{\prime 7} \beta^{(7)}\left(\mu^{\prime}-1\right) \rho\left[\left(\mu^{\prime}-1\right) \rho+2 \mu^{\prime} \tau\right] \\
& -\frac{1}{4} \mu^{\prime 7} \beta^{(3) 2}\left(\mu^{\prime}-1\right) \rho\left[\rho^{2}+3 \mu^{\prime} \tau^{2}\right]-\frac{1}{8} \mu^{\prime 5} \beta^{(5)}\left(\mu^{\prime}-1\right) \rho \\
& \cdot\left[\left(\mu^{\prime}+1\right)\left(\mu^{\prime 2}-1\right) \rho^{3}+4 \mu^{\prime 2} \tau\left(\rho^{2}+\mu^{\prime} \tau^{2}\right)\right]-\frac{1}{16} \mu^{\prime 3} \beta^{(3)}\left(\mu^{\prime}-1\right) \rho \cdot \\
& \cdot\left[\left(\mu^{\prime}+1\right)\left(\mu^{\prime 4}-1\right) \rho^{5}+2 \mu^{2}\left(\mu^{\prime 2}+\mu^{\prime}+1\right) \rho^{4} \tau+4 \mu^{\prime 4} \rho^{2} \tau^{3}+6 \mu^{\prime 5} \tau^{5}\right] \\
& +\frac{1}{128} \mu^{\prime}\left(\mu^{\prime}-1\right) \rho(\rho+\tau)\left(\rho-\mu^{\prime} \tau\right)\left[5\left(\mu^{\prime 6}+\mu^{\prime 5}+\mu^{\prime 4}+\mu^{\prime 3}+\mu^{\prime 2}+\mu^{\prime}+1\right) \rho^{6}\right. \\
& -\mu^{\prime}\left(\mu^{\prime}-1\right)\left(5 \mu^{\prime 4}+6 \mu^{\prime 3}+7 \mu^{\prime 2}+6 \mu^{\prime}+5\right) \rho^{5} \tau \\
& +\mu^{\prime 2}\left(5 \mu^{\prime 4}+\mu^{\prime 3}+3 \mu^{\prime 2}+\mu^{\prime}+5\right) \rho^{4} \tau^{2}-\mu^{\prime 3}\left(\mu^{\prime}-1\right)\left(5 \mu^{\prime 2}+4 \mu^{\prime}+5\right) \rho^{3} \tau^{3} \\
& \left.+\mu^{\prime 4}\left(5 \mu^{\prime 2}-\mu^{\prime}+5\right) \rho^{2} \tau^{4}-5 \mu^{\prime 5}\left(\mu^{\prime}-1\right) \rho \tau^{5}+5 \mu^{\prime 6} \tau^{6}\right]
\end{aligned}
$$

If preferred, $\left(\tau^{\prime}+\mu^{\prime} \tau\right)$ may be substitued for $\left[\left(\mu^{\prime}-1\right) \rho+2 \mu^{\prime} \tau\right]$ throughout these equations. In most numerical applications the computing of the higher powers of $\mu^{\prime}$ may be avoided by dividing each equation by the appropriate power of $n^{\prime}$, remembering that $\mu^{\prime}=\frac{n^{\prime}}{n}$. Equations (9) then take the following form:

$$
\begin{aligned}
& \frac{\tau^{\prime}}{n^{\prime}}=\frac{\tau}{n}+\left(\frac{1}{n}-\frac{1}{n^{\prime}}\right) \rho \\
& \frac{\beta^{\prime(3)}}{n^{\prime 3}}=\frac{\beta^{(3)}}{n^{3}}-\frac{1}{2} \rho\left[\left(\frac{1}{n}-\frac{1}{n^{\prime}}\right)^{2} \frac{\tau}{n} \rho+\frac{1}{n}\left(\frac{\rho^{2}}{n^{\prime 2}}+\frac{\tau^{2}}{n^{2}}\right)-\frac{1}{n^{\prime}}\left(\frac{\rho^{2}}{n^{2}}+\frac{\tau^{2}}{n^{2}}\right)\right] \\
& \frac{\beta^{\prime(5)}}{n^{\prime 5}}=\frac{\beta^{(5)}}{n^{5}}-\frac{1}{2} \frac{\beta^{(3)}}{n^{3}}\left(\frac{1}{n}-\frac{1}{n^{\prime}}\right) \rho\left[\left(\frac{1}{n}-\frac{1}{n^{\prime}}\right) \rho+2 \frac{\tau}{n}\right]-\frac{1}{8} \rho\left[\left(\frac{1}{n^{2}}-\frac{1}{n^{\prime 2}}\right)^{2} \frac{\tau}{n} \rho^{3}\right. \\
&\left.\quad+\frac{1}{n}\left(\frac{\rho^{2}}{n^{\prime 2}}-\frac{\tau^{2}}{n^{2}}\right)^{2}-\frac{1}{n^{\prime}}\left(\frac{\rho^{2}}{n^{2}}-\frac{\tau^{2}}{n^{2}}\right)^{2}\right] \\
& \frac{\beta^{\prime(7)}=}{n^{\prime 7}}=\frac{\beta^{(7)}}{n^{7}}-\frac{1}{2} \frac{\beta^{(3) 2}}{n^{6}}\left(\frac{1}{n}-\frac{1}{n^{\prime}}\right) \rho-\frac{1}{2} \frac{\beta^{(5)}}{n^{5}}\left(\frac{1}{n}-\frac{1}{n^{\prime}}\right) \rho\left[\left(\frac{1}{4}\left(\frac{1}{n^{2}}-\frac{1}{n^{\prime 2}}\right)^{2} \rho^{3}-\frac{1}{n} \frac{\tau}{n}\left(\frac{\rho^{2}}{n^{\prime 2}}-\frac{\tau^{2}}{n^{2}}\right)+\frac{1}{n^{\prime}} \frac{\tau}{n}\left(\frac{\rho^{2}}{n^{2}}-\frac{\tau^{2}}{n^{2}}\right)\right]\right. \\
& \quad-\frac{1}{16} \rho\left[\left(\frac{1}{n^{2}}-\frac{1}{n^{\prime 2}}\right)^{2}\left(\frac{1}{n^{2}}+\frac{1}{n^{\prime 2}}\right) \frac{\tau}{n} \rho^{5}+\frac{1}{n}\left(\frac{\rho^{2}}{n^{\prime 2}}-\frac{\tau^{2}}{n^{2}}\right)^{2}\left(\frac{\rho^{2}}{n^{\prime 2}}+\frac{\tau^{2}}{n^{2}}\right)\right. \\
&\left.\quad-\frac{1}{n^{\prime}}\left(\frac{\rho^{2}}{n^{2}}-\frac{\tau^{2}}{n^{2}}\right)^{2}\left(\frac{\rho^{2}}{n^{2}}+\frac{\tau^{2}}{n^{2}}\right)\right]
\end{aligned}
$$

If parallel light is incident upon a lens surface, $\tau=\beta^{(3)}=\beta^{(5)}=$ follows: $=0$. If also $n=1$ then equations (11) are greatly simplified, as

$$
\begin{aligned}
\frac{\tau^{\prime}}{n^{\prime}} & =\left(1-\frac{1}{n^{\prime}}\right) \rho \\
\frac{\beta^{\prime(3)}}{n^{\prime 3}} & =\frac{1}{2}\left(1-\frac{1}{n^{\prime}}\right) \rho \frac{\rho^{2}}{n^{\prime}} \\
\frac{\beta^{\prime(5)}}{n^{\prime 5}} & =\frac{1}{8}\left(1-\frac{1}{n^{\prime 3}}\right) \rho^{3} \frac{\rho^{2}}{n^{\prime}} \\
\frac{\beta^{\prime(7)}}{n^{\prime 7}} & =\frac{1}{16}\left(1-\frac{1}{n^{\prime 5}}\right) \rho^{5} \frac{\rho^{2}}{n^{\prime}}
\end{aligned}
$$


The higher-order equations in these last two sets may be written in a similar manner.

\section{DISCUSSION}

\section{(a) INHERENT AND PROPAGATED ABERRATION}

If the incident pencil is free from aberration, as is the case when it arises from a point on a material object, then $\beta^{(3)}=\beta^{(5)}=\ldots .=0$ and all terms in the aberration of the refracted pencil vanish except those in the upper row of Table 3; that is, all except the last term in each of the equations of (9), (10), or (11). This remaining term in each order will be referred to as the inherent aberration of that order, since it is independent of the aberration due to the other surfaces of the system and is entirely due to the surface in question. It is, of course, affected by a change of object distance.

If aberration is present in the incident pencil, then the terms of the image aberration which have a $\beta$ coefficient do not, in general, vanish. These terms are to be considered as representing the aberration due to the preceding surfaces of the system after its propagation through the surface under consideration, and will be designated in the following manner:

The term in the fifth order equation $((9 c),(10 c)$, or $(11 c))$ which contains the factor $\beta^{(3)}$ will be referred to as the third order term of the fifth order or the $\beta^{\prime(5)}\left(\beta^{(3)}\right)$ term. Similarly, the third order squared term of the seventh order denotes the $\beta^{\prime(7)}\left(\beta^{(3) 2}\right)$ term, and the third-fifth order term of the ninth order denotes the $\beta^{\prime(9)}\left(\beta^{(3)}\right.$ $\left.\beta^{(5)}\right)$ term, etc. These distinctions will apply directly to the system of equations to be developed later (Pt. III) except that the symbol $\zeta$ will replace $\beta$.

\section{(b) NOTES ON NUMERICAL APPLICATIONS}

There are a number of similarities among different terms of these equations which make the computation easier than would appear at first sight. The $\beta^{\prime(i)}\left(\beta^{(j)}\right)$ term is identical with the $\beta^{\prime(i+2)}\left(\beta^{(j+2)}\right)$ term, the $\beta^{\prime(i+4)}\left(\beta^{(j+4)}\right)$ term, etc., except for factors in $\mu^{\prime 2}$. Moreover, the $\beta^{\prime(i)}\left(\beta^{(3) 2}\right), \beta^{\prime(i+2)}\left(\beta^{(3)} \beta^{(5)}\right), \beta^{\prime(i+4)}\left(\beta^{(3)} \beta^{(7)}\right), \beta^{\prime(i+4)}\left(\beta^{(5) 2}\right)$, and the $\beta^{\prime(i+4)}\left(\beta^{(3) 3}\right)$ terms differ only by factors in $\mu^{\prime 2}, \frac{1}{2}$, and in one case $\tau$. $\quad(i$ and $j$ may be $3,5,7$, or $9 ; i \geqq j$.)

It is, of course, optional which form of the equations is to be used in computing. The third order of $(10 \mathrm{~b})$ is doubtless simpler than that of $(9 \mathrm{~b})$. However, if orders higher than the third are also to be computed, the equations (9) have their advantages, notably in the marked similarities among the following bracketed quantities:

$$
\begin{gathered}
{\left[\left(\mu^{\prime}-1\right)^{2} \rho \tau+\left(\rho^{2}+\mu^{\prime 2} \tau^{2}\right)-\mu^{\prime}\left(\rho^{2}+\tau^{2}\right)\right]} \\
{\left[\left(\mu^{\prime 2}-1\right)^{2} \rho^{3} \tau+\left(\rho^{2}-\mu^{\prime 2} \tau^{2}\right)^{2}-\mu^{\prime 3}\left(\rho^{2}-\tau^{2}\right)^{2}\right]} \\
{\left[\frac{1}{4}\left(\mu^{\prime 2}-1\right)^{2} \rho^{3}-\mu^{\prime 2} \tau\left(\rho^{2}-\mu^{\prime 2} \tau^{2}\right)+\mu^{\prime 3} \tau\left(\rho^{2}-\tau^{2}\right)\right]} \\
{\left[\left(\mu^{\prime 2}-1\right)^{2}\left(\mu^{\prime 2}+1\right) \rho^{5} \tau+\left(\rho^{2}-\mu^{\prime 2} \tau^{2}\right)^{2}\left(\rho^{2}+\mu^{\prime 2} \tau^{2}\right)-\mu^{\prime 5}\left(\rho^{2}-\tau^{2}\right)^{2}\left(\rho^{2}+\tau^{2}\right)\right]}
\end{gathered}
$$


Each of these quantities is the sum of three terms. Each term in the last bracket (seventh order) contains as factors the corresponding terms in the first two brackets, except for $\left(\mu^{\prime 2}+1\right)$ instead of $\left(\mu^{\prime}-1\right)^{2}$. Also the terms in the third bracket contain factors of the terms in the second bracket. All this reduces considerably the labor involved in

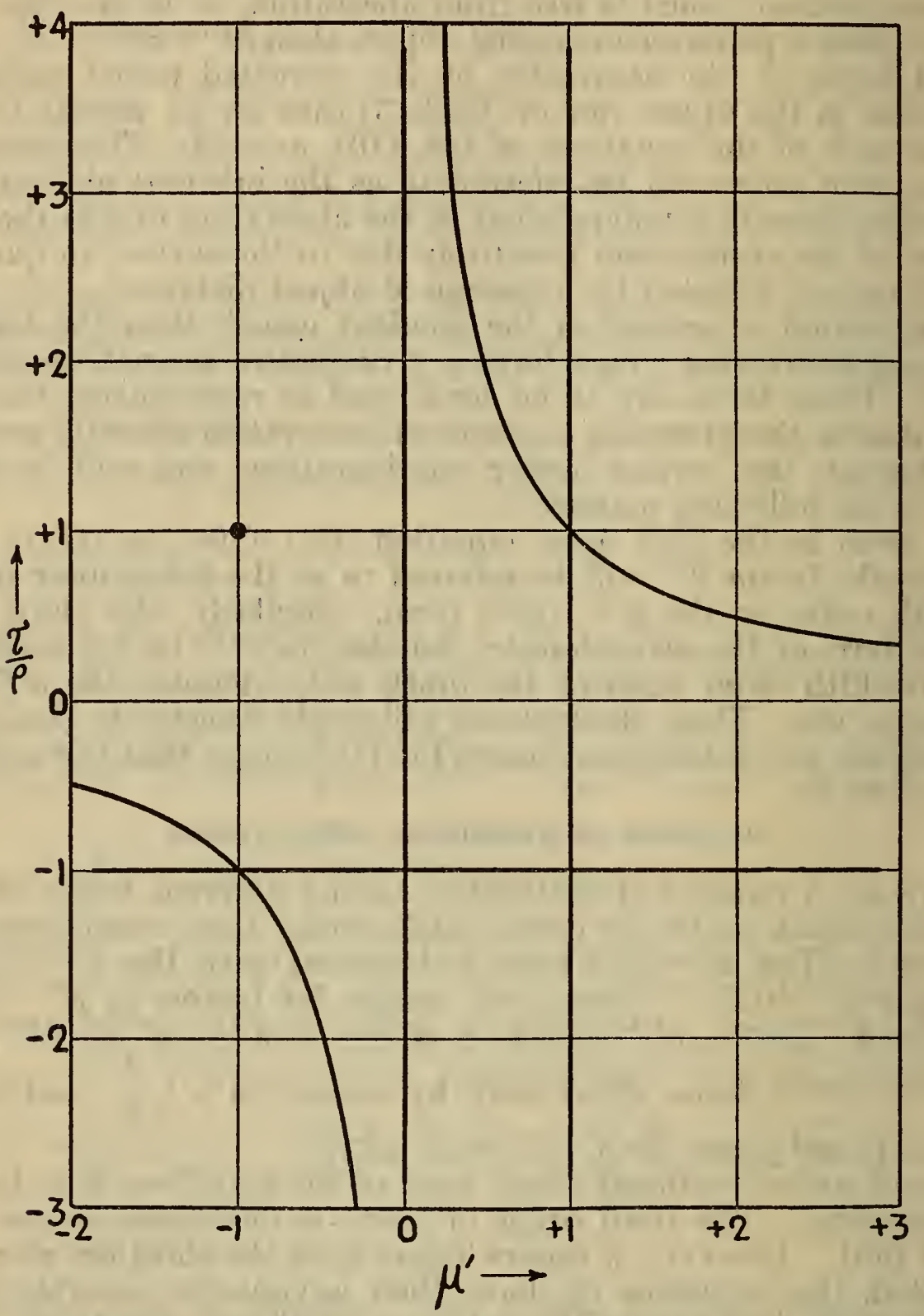

Figure 3.-Zero reciprocal spherical aberration contour

The point at $\mu^{\prime}=-1, \frac{r}{\rho}=+1$ pertains only to orders higher than the third.

computing. Either of these sets may be written in the reduced form, as equations (11).

(c) ROOTS, ANOMALOUS POINTS, AND SPECIFIC EXAMPLES

It is interesting to note the conditions under which the aberration of a surface vanishes; that is, to find the root of the inherent terms 
f the equations (9) or (10) when set equal to zero. In equations (10) he factors common to all orders may be written thus:

$$
\rho^{3}\left[\mu^{\prime}\left(\mu^{\prime}-1\right)\left(1+\frac{\tau}{\rho}\right)\left(1-\mu^{\prime} \frac{\tau}{\rho}\right)\right]=0
$$

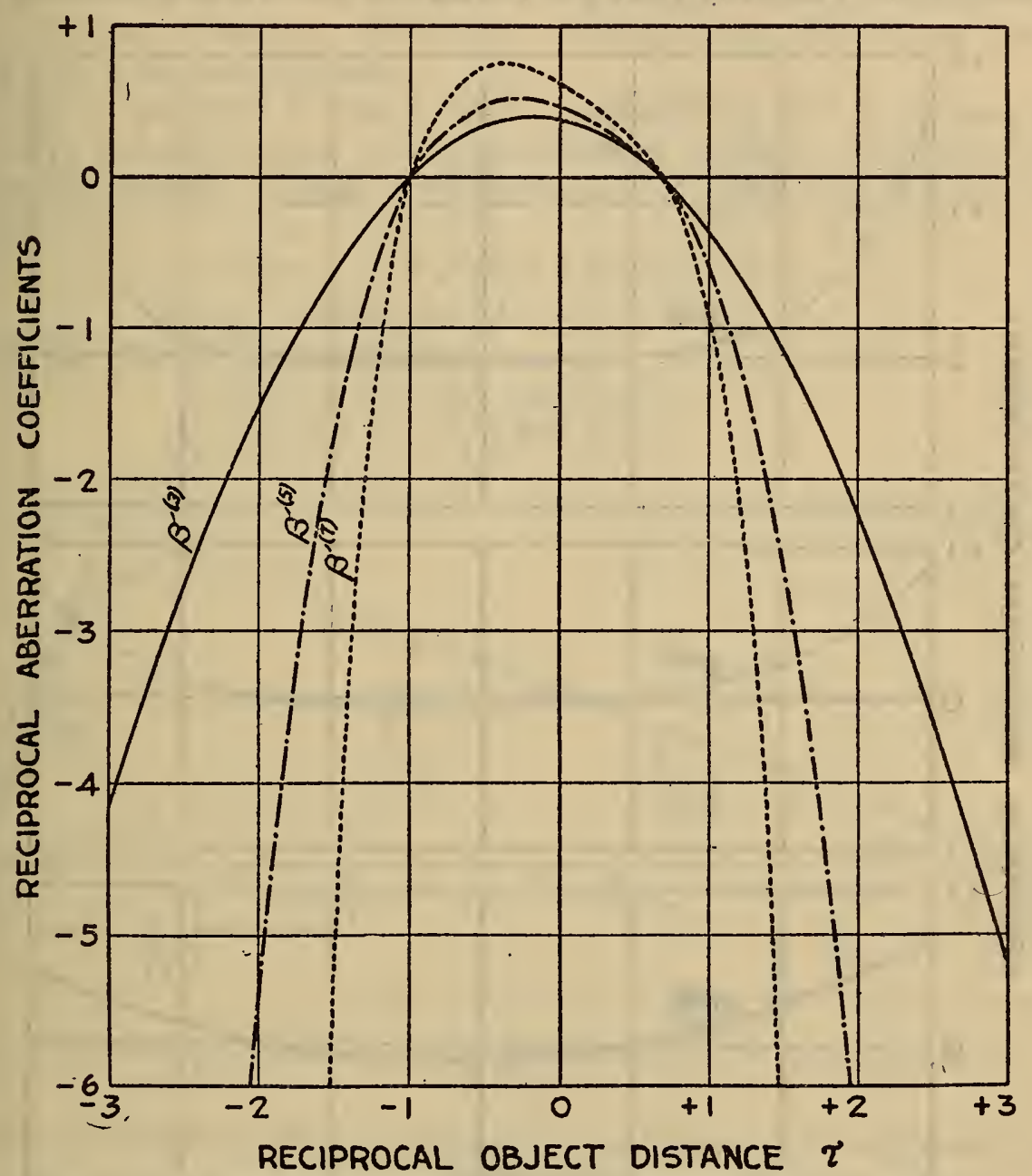

FIGURE 4.-Reciprocal aberration coefficients for surface of unit radius, $\mu^{\prime}=1.5$

When $\rho$ has a finite value not zero the roots of this are as follows:

$\mu^{\prime}=0$ Physically impossible.

$\mu^{\prime}=1$ Same index on both sides; no refraction.

$\tau / \rho=-1$

or

Object and image coincide at the vertex.

$\tau=-\rho$

$\mu^{\prime} \frac{\tau}{\rho}=1$

$$
\begin{aligned}
& \rho=\rho / \mu^{\prime} \\
& \rho
\end{aligned} \text { The well-known aplanatic point. }
$$

These roots are shown in Figure 3 . If $\rho=0$, the surface is a plane and there is no center of curvature from which to measure the distances. If the object coincides with the center of curvature then 
$\tau=\infty$ and the series is nonconvergent. (See Part VII, sec. 1.) Another set of formulas will be derived which may be used in these cases.

The orders higher than the third have the additional roots given by equating to zero the quantities in brackets in the inherent terms of equations (10). The only root yet found for these is the isolated point

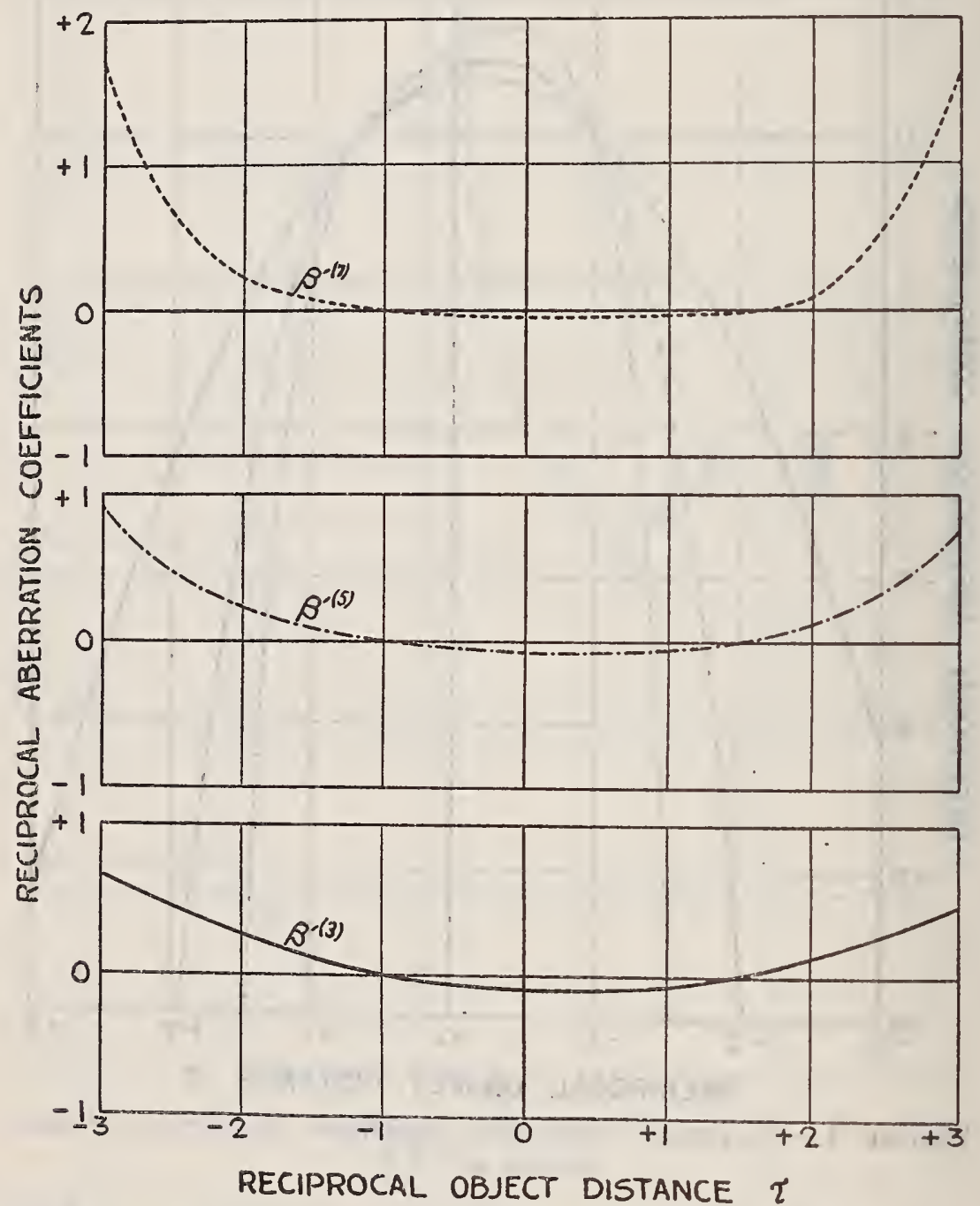
FIgURE 5.-Reciprocal aberration coefficients for surface of unit
radius, $\mu^{\prime}=1 / 1.5$

$\left(\frac{\tau}{\rho}=+1, \mu^{\prime}=-1\right)$ indicated in Figure 3. Since the value $\mu^{\prime}=-1$ indicates a reflection, it may be noted that in the image formed by a spherical mirror the reciprocal aberration of all orders higher than the third vanishes when the object is at a distance $2 r$ from the vertex.

The advisability of computing and tabulating the values of these coefficients for different values of $\mu^{\prime}$ and $\tau$ is being considered. Figures 4 and 5 show, for the values $\mu^{\prime}=1.5$ and $\mu^{\prime}=1 / 1.5$, the form of the functions which would result. These values would have to be multiplied by the power of $\rho$ corresponding to the order of the aberration to obtain the actual value of any particular coefficient. 


\section{TRANSFER FORMULAS}

\section{DERIVATION}

The refracted or image pencil from one surface is, of course, identical with the incident or object pencil of the succeeding surface. However, when the point of reference is changed, the aberration coefficients are also altered. Formulas will now be developed for computing the new coefficients.

Let the subscripts $i$ and $j$ refer, respectively, to a surface whose aberration is known and to the succeeding surface, whose aberration is to be computed. Then, as before, see $\left(2^{\prime}\right)$ and $\left(3^{\prime}\right)$ for the known surface,

$$
\Theta^{\prime}{ }_{i}=\tau^{\prime}{ }_{i}+\beta^{\prime}{ }_{i}{ }^{(3)} h^{\prime}{ }_{i}{ }^{2}+\beta^{\prime}{ }_{i}{ }^{(5)} h^{\prime}{ }_{i}{ }^{4}+\ldots
$$

$$
\sin \alpha^{\prime}{ }_{i}=\theta^{\prime}{ }_{i} h_{i}^{\prime}=\tau^{\prime}{ }_{i} h_{i}^{\prime}+\beta^{\prime}{ }_{i}{ }^{(3)} h^{\prime}{ }_{i}{ }^{3}+\ldots .
$$

and for the succeeding surface,

$$
\begin{aligned}
\theta_{j} & =\tau_{j}+\beta_{j}{ }^{(3)} h_{j}{ }^{2}+\beta_{j}{ }^{(5)} h_{j}{ }^{4}+\ldots \\
\sin \alpha_{j} & =\theta_{j} h_{j}=\tau_{j} h_{j}+\beta^{(3)}{ }_{j} h^{3}{ }_{j}+\ldots
\end{aligned}
$$

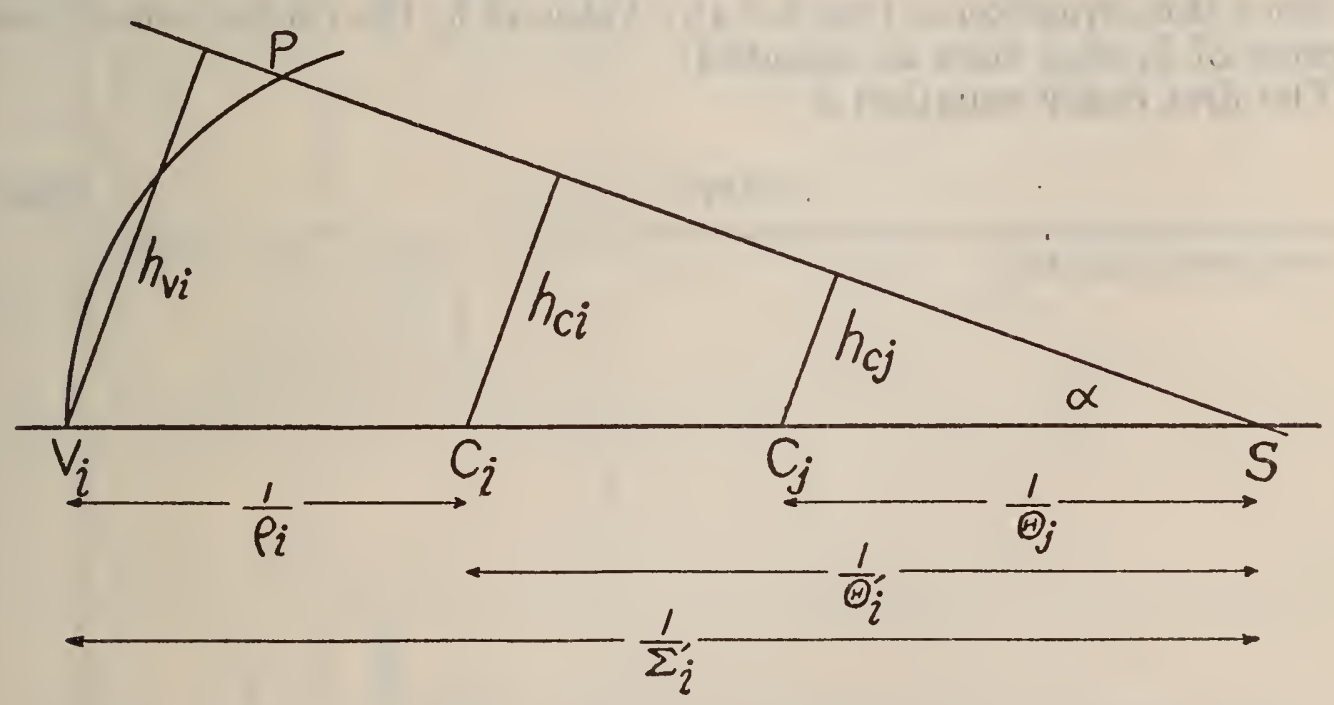

FIgURE 6.-Diagram showing aperture, $h$, as measured from different points on the axis

Let $C_{i}$ and $C_{j}$ (fig. 6 ) be the respective centers of curvature, and let the distance from $C_{i}$ to $C_{j}$ be $d$, positive if to the right. Then, for any ray, as $P S$, the adding of axial distances gives:

$$
\frac{1}{\theta^{\prime}}=\frac{1}{\theta_{j}}+d
$$

Similarly, for a paraxial ray

then:

$$
\frac{1}{\tau^{\prime}}=\frac{1}{\tau_{j}}+d, \text { or } \frac{1}{\tau_{j}}=\frac{1}{\tau_{i}^{\prime}}-d
$$

$$
\frac{\tau_{j}}{\tau^{\prime}{ }_{i}}=1+d \tau_{j}=\frac{1}{1-d \tau^{\prime}{ }_{i}}=\kappa
$$

where $\kappa$ is a new symbol introduced for convenience.

Manifestly

$$
\sin \alpha^{\prime}{ }_{i}=\sin \alpha_{\jmath}
$$


or, by equations (13) and (15):

$$
h_{i}^{\prime}=\frac{1}{\theta_{i}^{\prime}} \theta_{j} h_{j}
$$

Then, substituting in turn from equations (16), (14), and (18):

$$
\begin{aligned}
h^{\prime}{ }_{i} & =\left(1+d \Theta_{j}\right) h_{j} \\
& =\left(1+d \tau_{j}\right) h_{j}+d \beta_{j}{ }^{(3)} h^{3}{ }_{j}+\ldots \\
& =\kappa h_{j}+d \beta_{j}{ }^{(3)} h^{3}{ }_{j}+d \beta_{j}{ }^{\left({ }^{3}\right)} h^{5}{ }_{j}+\ldots
\end{aligned}
$$

This last expression for $h^{\prime}{ }_{i}$ may be substituted in the right-hand member of equation (13), and then since the latter is equal to equation (15)

$$
\begin{aligned}
\sin \alpha_{j} & =\tau_{j} h_{j}+\beta_{j}{ }^{(3)} h^{3}{ }_{j}+\beta_{j}{ }^{(5)} h^{5}{ }_{j}+\ldots \\
& =\text { the series expanded }{ }^{16} \text { in Table } 4 .
\end{aligned}
$$

Since this equation is true for any value of $h_{f}$ the coefficients of like powers of $h_{f}$ may then be equated.

The first order equation is

$$
\tau_{j}=\kappa \tau^{\prime}{ }_{i}
$$

16 See footnote 15, p. 196. 


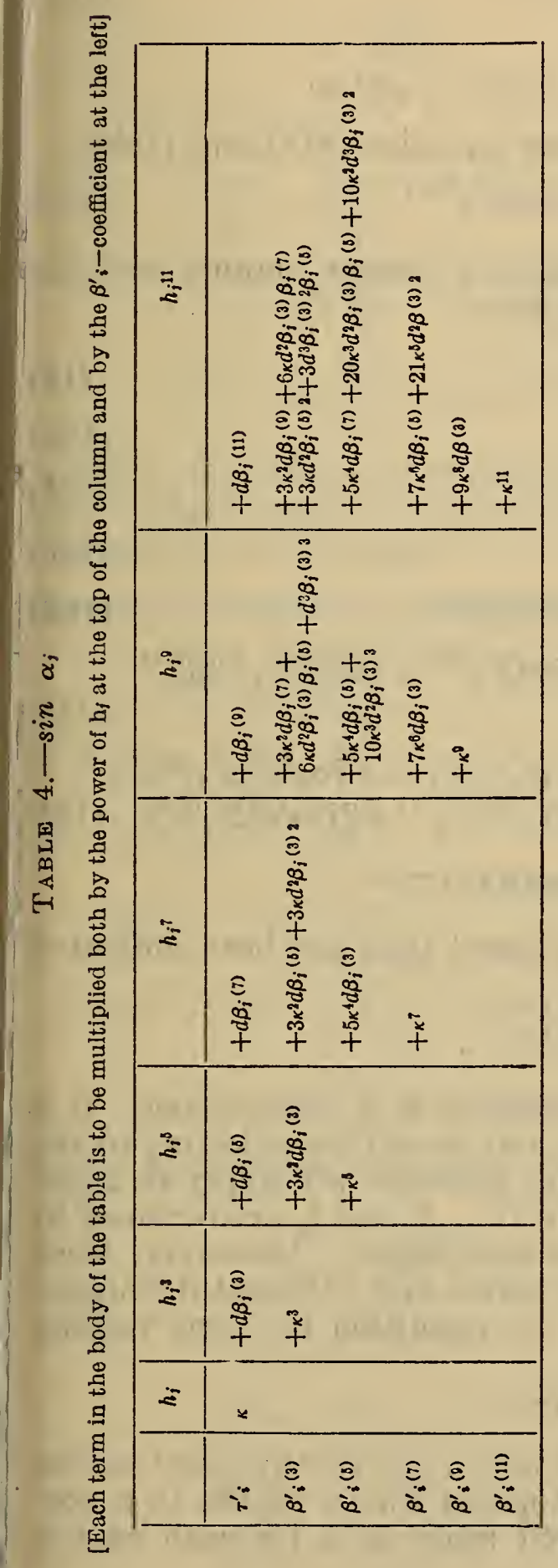


The third order, after combining terms, is

$$
\left(1-d{\tau^{\prime}}_{i}\right) \beta_{j}{ }^{(3)}=\kappa^{3} \beta^{\prime}{ }_{i}^{(3)}
$$

or, substituting from equation (18)

$$
\beta_{j}{ }^{(3)}=\kappa^{4} \beta^{\prime}{ }_{i}{ }^{(3)}
$$

The fifth order, likewise, is

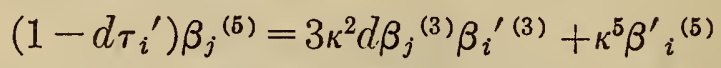

Substituting for $\left(1-d \tau_{i}{ }^{\prime}\right)$ and $\beta_{j}{ }^{(3)}$ from equations (18) and $(18 b)$ :

$$
\beta_{j}{ }^{(5)}=\kappa^{6}\left(\beta^{\prime}{ }_{i}{ }^{(5)}+3 \kappa d \beta^{\prime}{ }_{i}{ }^{(3) 2}\right)
$$

The higher orders may be reduced in a similar manner and the transfer formulas summarized as follows:

$$
\begin{aligned}
& \kappa=\frac{1}{1-d \tau^{\prime}} \\
& \tau_{j}=\kappa \tau^{\prime}{ }_{i} \\
& \beta_{j}{ }^{(3)}=\kappa^{4} \beta^{\prime}{ }_{i}{ }^{(3)} \\
& \beta_{j}{ }^{(5)}=\kappa^{6}\left(\beta^{\prime}{ }_{i}{ }^{(5)}+3 \kappa d \beta^{\prime}{ }_{i}{ }^{(3) 2}\right) \\
& \beta_{j}{ }^{(7)}=\kappa^{8}\left(\beta^{\prime}{ }_{i}{ }^{(7)}+8 \kappa d \beta^{\prime}{ }_{i}{ }^{(3)} \beta^{\prime(5)}+12 \kappa^{2} d^{2} \beta^{\prime}{ }_{i}{ }^{(3) 3}\right)
\end{aligned}
$$

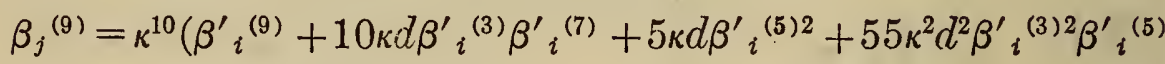

$$
\begin{aligned}
& \left.+55 \kappa^{3} d^{3} \beta^{\prime}{ }_{i}^{(3) .4}\right) \\
& \beta_{j}{ }^{(11)}=\kappa^{12}\left[\beta^{\prime}{ }_{i}{ }^{(11)}+12 \kappa d\left({\beta^{\prime}}_{i}{ }^{(3)}{\beta^{\prime}}^{\prime}{ }_{i}{ }^{(9)}+{\beta^{\prime}}^{\prime}{ }_{i}{ }^{(5)}{\beta^{\prime}}_{i}{ }^{(7)}\right)+78 \kappa^{2} d^{2}\left(\beta^{\prime}{ }_{i}{ }^{(3) 2}{ }^{\prime}{ }_{i}{ }_{i}{ }^{(7)}\right.\right. \\
& \left.\left.+\beta^{\prime}{ }_{i}{ }^{(3)} \beta^{\prime}{ }_{i}{ }^{(5) 2}\right)+364 \kappa^{3} d^{3}{\beta^{\prime}}^{\prime}{ }_{i}{ }^{(3) 3} \beta^{\prime}{ }_{i}{ }^{(5)}+273 \kappa^{4} d^{4} \beta^{\prime}{ }_{i}{ }^{(3) 5}\right]
\end{aligned}
$$

\section{NOTES ON GENERALITY}

These transfer formulas are more general than has been indicated By definition, the series

$$
\Theta=\tau+\beta^{(3)} h_{c}^{2}+\beta^{(5)} h_{c}^{4}+\ldots
$$

refers exclusively to the center of curvature of a lens surface. It is evident, however, that a similar series may be written referring to any point on the axis and that the transfer formulas will apply in transferring to any other point on the axis if $\theta, \tau, \beta$, and $h_{c}$ are replaced by the corresponding symbols from the new series. Moreover, these formulas apply as well to some of the series with different definitions of $h$ mentioned in the introduction, the condition for their validity being

$$
\Psi h=f(\alpha)
$$

where $\Psi$ corresponds to $\theta$ above, but may refer to any point on the axis and $f(\alpha)$ is any function of $\alpha$ the use of which results in a convergent series throughout the required range of $h$ for each case in question. 
3. TRANSFER FORMULAS INVOLVING POWERS OF $n$

If the reduced form of the aberration equations (equations (11) or (12)) is used, then the transfer formulas must also be divided by ihe corresponding powers of $n$. They are then as follows:

$$
\begin{aligned}
& \kappa=\frac{1}{1-n_{i}^{\prime} d \frac{\tau_{i}^{\prime}}{n_{i}^{\prime}}} \\
& \begin{array}{c}
\tau_{j}^{j} \\
n_{j}
\end{array}=\kappa \frac{\tau_{i}^{\prime}}{n_{i}^{\prime}} \\
& \frac{\beta_{i}^{(3)}}{n_{i}^{3}}=\kappa^{4} \frac{\beta_{i}^{\prime(3)}}{n_{i}^{3}} \\
& \frac{\beta_{i}^{(5)}}{n_{i}^{5}}=\kappa^{6}\left(\frac{\beta_{i}^{\prime(5)}}{n_{i}^{\prime 5}}+3 \kappa n_{i}^{\prime} d \frac{\beta_{i}^{\prime(3) 2}}{n_{i}^{\prime 6}}\right) \\
& \frac{\beta_{j}^{(7)}}{n_{i}^{7}}=\kappa^{8}\left(\frac{\beta_{i}^{\prime}(7)}{n_{i}^{7}}+8 \kappa n_{i}^{\prime} d \frac{\beta_{i}^{\prime}{ }_{i}^{(3)} \beta_{i}^{\prime}{ }^{(5)}}{n_{i}^{\prime 8}}+12 \kappa^{2} n_{i}^{\prime 2} d^{2} \frac{\beta_{i}^{\prime(3) 3}}{n_{i}^{\prime 9}}\right)
\end{aligned}
$$

Other reduced forms of aberration equations are to be given later. The various forms of the transfer formulas are collected here for convenience in reference.

Center of curvature to vertex:

$$
\begin{aligned}
& n \kappa=\frac{1}{\frac{1}{n}-d \frac{\tau_{i}^{\prime}}{n_{i}^{\prime}}} \\
& n_{j} \sigma_{j}=n(n \kappa) \frac{\tau_{i}{ }^{\prime}}{n_{i}^{\prime}} \\
& n_{j} \zeta_{j}^{(3)}=(n \kappa)^{\dot{1}} \frac{\beta_{i}{ }^{(3)}}{n_{i}{ }^{\prime 3}} \\
& n_{j} \zeta_{j}{ }^{(5)}=(n \kappa)^{6}\left[\frac{\beta_{i}{ }^{(5)}}{n_{i}{ }^{(5}}+3(n \kappa) d \frac{\beta_{i}{ }^{(3) 2}}{n_{i}{ }^{\prime 6}}\right] \\
& n_{j} \zeta_{j}{ }^{(7)}=(n \kappa)^{8}\left[\frac{\beta_{i}{ }^{\prime(7)}}{n_{i}{ }^{\prime 7}}+8(n \kappa) d \frac{\beta^{\prime}{ }_{i}^{(3)} \beta_{i}{ }^{\prime(5)}}{n_{i}{ }^{8}}+12(n \kappa d)^{2} \frac{\beta_{i}{ }^{(3) 3}}{n_{i}{ }^{\prime 9}}\right]
\end{aligned}
$$

Vertex to center of curvature:

$$
\begin{aligned}
\frac{\kappa}{n}= & \frac{1}{n-d n_{i}{ }^{\prime} \sigma_{i}{ }^{\prime}} \\
\frac{\tau_{j}}{n_{j}}= & \frac{1}{n} \frac{\kappa}{n} n_{i}{ }^{\prime} \sigma_{i}{ }^{\prime} \\
\frac{\beta_{j}{ }^{(3)}}{n_{j}{ }^{3}}= & \left(\frac{\kappa}{n}\right)^{4} n_{i}{ }^{\prime} \zeta_{i}{ }^{\prime(3)} \\
\frac{\beta_{j}{ }^{(5)}}{n_{j}{ }^{5}}= & \left(\frac{\kappa}{n}\right)^{8}\left[n_{i}{ }^{\prime} \zeta_{i}{ }^{(5)}+3 \frac{\kappa}{n} d\left(n_{i}{ }^{\prime} \zeta_{i}{ }^{\prime(3)}\right)^{2}\right] \\
\frac{\beta_{j}{ }^{(7)}}{n_{j}{ }^{7}}= & \left(\frac{\kappa}{n}\right)^{8}\left[n_{i}{ }^{\prime} \zeta_{i}{ }^{(7)}+8 \frac{\kappa}{n} d\left(n_{i}{ }^{\prime} \zeta_{i}{ }^{\prime(3)}\right)\left(n_{i}{ }^{\prime} \zeta_{i}{ }^{\prime(5)}\right)\right. \\
& \left.+12\left(\frac{\kappa}{n} d\right)^{2}\left(n_{i}{ }^{\prime} \zeta_{i}{ }^{\prime(3)}\right)^{3}\right]
\end{aligned}
$$


Vertex to vertex:

$$
\begin{aligned}
\kappa= & \frac{1}{1-\frac{d}{n} n_{i}{ }^{\prime} \sigma_{i}{ }^{\prime}} \\
n_{j} \sigma_{f}= & \kappa n_{i}{ }^{\prime} \sigma_{i}{ }^{\prime} \\
n_{j} \zeta_{j}{ }^{\prime(3)}= & \kappa^{4} n_{i}{ }^{\prime} \zeta^{\prime}{ }^{(3)} \\
n_{j} \zeta_{j}{ }^{(5)}= & \kappa^{6}\left[n_{i}{ }^{\prime} \zeta_{i}{ }^{(5)}+3 \kappa \frac{d}{n}\left(n_{i}{ }^{\prime} \zeta_{i}{ }^{(3)}\right)^{2}\right] \\
n_{j} \zeta_{j}{ }^{(7)}= & \kappa^{8}\left[n_{i}{ }^{\prime} \zeta_{i}{ }^{(7)}+8 \kappa \frac{d}{n}\left(n_{i}{ }^{\prime} \zeta_{i}{ }^{\prime(3)}\right)\left(n_{i}{ }^{\prime} \zeta_{i}{ }^{(5)}\right)\right. \\
& \left.\quad+12\left(\kappa \frac{d}{n}\right)^{2}\left(n_{i}{ }^{\prime} \zeta_{i}{ }^{(3)}\right)^{3}\right]
\end{aligned}
$$

Higher orders are derived from equations $(18 e)$ and (18f) in a similar manner.

\section{THE RECIPROCAL ABERRATION OF A SINGLE SURFACE REFERRED TO THE VERTEX}

A second set of formulas will now be investigated in which the point of reference is chosen at the vertex of the surface. These are to be used in the cases where the formulas referred to the center of curvature are not applicable (see p. 203), although in many cases either set may be used.

\section{DERIVATION OF THE FORMULAS}

As has been stated, aberration series of the form (2) or $\left(2^{\prime}\right)$ may be written referring to any point on the axis. Let the two which refer to the vertex of a surface (as $V_{i}$, fig. 6 ) be

$$
\Sigma=\sigma+\zeta^{(3)} h_{v}{ }^{2}+\zeta^{(5)} h_{v}{ }^{4}+\zeta^{(7)} h_{v}{ }^{6}+\ldots
$$

for the incident pencil and

$$
\Sigma^{\prime}=\sigma^{\prime}+\zeta^{\prime(3)} h^{\prime}{ }_{0}{ }^{2}+\zeta^{\prime(5)} h^{\prime}{ }_{0}^{4}+\zeta^{\prime(7)} h^{\prime}{ }_{0}^{6}+\ldots
$$

for the refracted pencil. Probably the easiest way to establish a relation between these two series is to transfer the aberration to the center of curvature by means of equations (18) so that the resulting coefficients are identical with those of series $(2)$ and $\left(2^{\prime}\right)$ and then to substitute these coefficients in equations (10).

Let $\kappa$ and $\kappa^{\prime}$ be the constants of transfer in the object and image spaces, respectively. Then substituting the appropriate values for 
the object space in the formulas (18), the following relations are derived:

$$
\begin{aligned}
\kappa & =\frac{1}{1-r \sigma}=1+r \tau=\frac{\rho}{\rho-\sigma}=\frac{\rho+\tau}{\rho} \\
\tau & =\kappa \sigma \\
\beta^{(3)} & =\kappa^{4} \zeta^{(3)} \\
\beta^{(5)} & =\kappa^{6}\left(\zeta^{(5)}+3 \kappa r \zeta^{(3) 2}\right) \\
\beta^{(7)} & =\kappa^{8}\left(\zeta^{(7)}+8 \kappa r \zeta^{(3)} \zeta^{(5)}+12 \kappa^{2} r^{2} \zeta^{(3) 3}\right) \\
\beta^{(9)} & =\kappa^{10}\left(\zeta^{(9)}+10 \kappa r \zeta^{(3)} \zeta^{(7)}+5 \kappa r \zeta^{(5) 2}+55 \kappa^{2} r^{2} \zeta^{(3) 2} \zeta^{(5)}+55 \kappa^{3} r^{3} \zeta^{(3) 4}\right)
\end{aligned}
$$

By a similar substitution and also by reason of equations (10a) and (24)

$$
\kappa^{\prime}=\frac{\rho}{\rho-\sigma}=\frac{\rho+\tau}{\rho}=\frac{\mu^{\prime}(\rho+\tau)}{\rho}=\mu^{\prime} \kappa
$$

in the case of the refracted pencil. Continuing the substitution

$$
\begin{aligned}
\tau^{\prime} & =\kappa^{\prime} \sigma^{\prime}=\mu^{\prime} \kappa \sigma^{\prime} \\
\beta^{\prime(3)} & =\mu^{\prime 4} \kappa^{4} \zeta^{\prime(3)}
\end{aligned}
$$

and similarly the higher orders differ in form from (24c to $24 e$ ) only by the use of $\mu^{\prime} \kappa$ instead of $\kappa$.

Substituting these values throughout equations (10), dividing each equation by the power of $\mu^{\prime} \kappa$ appearing as a factor of the left member, and writing $\frac{1}{\mu^{\prime}}=\frac{n}{n^{\prime}}=\mu$

$$
\begin{aligned}
\sigma^{\prime}= & \left(\sigma+\frac{\rho}{\kappa}\right)-\mu_{\kappa}^{\frac{\rho}{\kappa}} \\
\zeta^{\prime(3)}= & \mu \zeta^{(3)}+\frac{1}{2} \mu(1-\mu) \frac{\rho}{\kappa^{2}}\left(\frac{\rho}{\kappa}+\sigma\right)\left(\mu \frac{\rho}{\kappa}-\sigma\right) \\
\left(\zeta^{\prime(5)}+\right. & \left.3 \mu^{\prime} \kappa r \zeta^{\prime(3) 2}\right) \\
= & \mu\left(\zeta^{(5)}+3 \kappa r \zeta^{(3) 2}\right)-\frac{1}{2} \mu \zeta^{(3)}(1-\mu) \frac{\rho}{\kappa} \cdot \\
& \cdot\left[(1-\mu) \frac{\rho}{\kappa}+2 \sigma\right]+\frac{1}{8} \mu(1-\mu) \frac{\rho}{\kappa^{2}}\left(\frac{\rho}{\kappa}+\sigma\right)\left(\mu \frac{\rho}{\kappa}-\sigma\right) \\
& \cdot\left[\left(1+\mu+\mu^{2}\right) \frac{\rho^{2}}{\kappa^{2}}-(1-\mu) \frac{\rho}{\kappa} \sigma+\sigma^{2}\right] \\
\left(\zeta^{\prime(7)}+\right. & \left.8 \mu^{\prime} \kappa r \zeta^{\prime(3)} \zeta^{\prime(5)}+12 \mu^{\prime 2} \kappa^{2} r^{2} \zeta^{\prime(3) 3}\right) \\
= & \mu\left(\zeta^{(7)}+8 \kappa r \zeta^{(3)} \zeta^{(5)}+12 \kappa^{2} r^{2} \zeta^{(3) 3}\right)-\frac{1}{2} \mu \zeta^{(3) 2}(1-\mu) \rho \\
- & \frac{1}{2} \mu\left(\zeta^{(5)}+3 \kappa r \zeta^{(3) 2}\right)(1-\mu) \frac{\rho}{\kappa}\left[(1-\mu) \frac{\rho}{\kappa}+2 \sigma\right] \\
- & \frac{1}{8} \mu \zeta^{(3)}(1-\mu) \frac{\rho}{\kappa}\left[\left(1+\mu+\mu^{2}+\mu^{3}\right) \frac{\rho^{3}}{\kappa^{3}}+4 \mu \frac{\rho^{2}}{\kappa^{2}} \sigma+4 \sigma^{3}\right] \\
+ & \frac{1}{16} \mu(1-\mu) \frac{\rho}{\kappa^{2}}\left(\frac{\rho}{\kappa}+\sigma\right)\left(\mu \frac{\rho}{\kappa}-\sigma\right)\left[\left(1+\mu+\mu^{2}+\mu^{3}+\mu^{4}\right) \frac{\rho^{4}}{\kappa^{4}}\right. \\
- & \left.\left(1-\mu^{3}\right) \frac{\rho^{3}}{\kappa^{3}} \sigma+\left(1+\mu^{2}\right) \frac{\rho^{2}}{\kappa^{2}} \sigma^{2}-(1-\mu) \frac{\rho}{\kappa} \sigma^{3}+\sigma^{4}\right]
\end{aligned}
$$




$$
\begin{aligned}
\left(\zeta^{\prime(9)}\right. & +10 \mu^{\prime} \kappa r \zeta^{\prime(3)} \zeta^{\prime(7)}+5 \mu^{\prime} \kappa r \zeta^{\prime(5) 2} \\
& \left.+55 \mu^{\prime 2} \kappa^{2} r^{2} \zeta^{(3) 2} \zeta^{\prime(5)}+55 \mu^{\prime 3} \kappa^{3} r^{3} \zeta^{\prime(3) 4}\right) \\
& =\mu\left(\zeta^{(9)}+10 \kappa r \zeta^{(3)} \zeta^{(7)}+5 \kappa r \zeta^{(5) 2}+55 \kappa^{2} r^{2} \zeta^{(3) 2} \zeta^{(5)}\right. \\
& \left.+55 \kappa^{3} r^{3} \zeta^{(3) 4}\right)-\mu\left(\zeta^{(3)} \zeta^{(5)}+3 \kappa r \zeta^{(3) 3}\right)(1-\mu)-\frac{1}{2} \mu \\
& \cdot\left(\zeta^{(7)}+8 \kappa r \zeta^{(3)} \zeta^{(5)}+12 \kappa^{2} r^{2} \zeta^{(3) 3}\right)(1-\mu) \frac{\rho}{\kappa}\left[(1-\mu) \frac{\rho}{\kappa}+2 \sigma\right] \\
& -\frac{1}{4} \mu \zeta^{(3) 2}(1-\mu) \rho\left[\mu \frac{\rho^{2}}{\kappa^{2}}+3 \sigma^{?}\right]-\frac{1}{\delta} \mu\left(\zeta^{(5)}+3 \kappa r \zeta^{(3) 2}\right)(1-\mu) \frac{\rho}{\kappa} . \\
& +\left[\left(1+\mu-\mu^{2}-\mu^{3}\right) \frac{\rho^{3}}{\kappa^{3}}+4 \mu \frac{\rho^{2}}{\kappa^{2}} \sigma+4 \sigma^{3}\right]-\frac{1}{16} \mu \zeta^{(3)}(1-\mu) \frac{\rho}{\kappa} \cdot \\
& +\left[\left(1+\mu-\mu^{4}-\mu^{5}\right) \frac{\rho^{5}}{\kappa^{5}}+2\left(\mu+\mu^{2}+\mu^{3}\right) \frac{\rho^{4}}{\kappa^{4}} \sigma+4 \mu \frac{\rho^{2}}{\kappa^{2}} \sigma^{3}+6 \sigma^{5}\right] \\
& +\frac{1}{128} \mu(1-\mu) \frac{\rho}{\kappa^{2}}\left(\frac{\rho}{\kappa}+\sigma\right)\left(\mu \frac{\rho}{\kappa}-\sigma\right)\left[5\left(1+\mu+\mu^{2}+\mu^{3}+\mu^{4}+\mu^{5}+\mu^{6}\right) \frac{\rho^{6}}{\kappa^{6}}\right. \\
& -\left(5+\mu+\mu^{2}-\mu^{3}-\mu^{4}-\mu^{5}\right) \frac{\rho^{5}}{\kappa^{5}} \sigma+\left(5+\mu+3 \mu^{2}+\mu^{3}+5 \mu^{4}\right) \frac{\rho^{4}}{\kappa^{4}} \sigma^{2} \\
& \left.-\left(5-\mu+\mu^{2}-5 \mu^{3}\right) \frac{\rho^{3}}{\kappa^{3}} \sigma^{3}+\left(5-\mu+5 \mu^{2}\right) \frac{\rho^{2}}{\kappa^{2}} \sigma^{4}-(5-5 \mu) \frac{\rho}{\kappa} \sigma^{5}+5 \sigma^{6}\right]
\end{aligned}
$$

Equation (26b) is used in eliminating $\zeta^{\prime(3)}$ from equations $(26 c)$ to $(26 e)$. Following this step, the resulting form of equation $(26 c)$ is used in eliminating $\zeta^{\prime(5)}$ from the higher order equations, and so on. Remembering at the same time that, from equation $(24), \frac{\rho}{\kappa}=(\rho-\sigma)$ and $\left(\frac{\rho}{\kappa}+\sigma\right)=\rho$. The equations may be brought into the following form:

$$
\begin{aligned}
\sigma^{\prime}= & \rho-\mu(\rho-\sigma)=\mu \sigma-(\mu-1) \rho \\
\zeta^{\prime(3)}= & \mu \zeta^{(3)}-\frac{1}{2} \mu(\mu-1)(\rho-\sigma)^{2}[\mu(\rho-\sigma)-\sigma] \\
\zeta^{\prime(5)}= & \mu \zeta^{(5)}+\frac{1}{2} \mu \zeta^{(3)}(\mu-1)(\rho-\sigma)[(5 \mu+1)(\rho-\sigma)-4 \sigma]-\frac{1}{8} \mu(\mu-1) \\
& \quad(\rho-\sigma)^{2}[\mu(\rho-\sigma)-\sigma]\left[\left(7 \mu^{2}-5 \mu+1\right)(\rho-\sigma)^{2}-(5 \mu-5)\right. \\
& \left.\quad(\rho-\sigma) \sigma+\sigma^{2}\right] \\
\zeta^{\prime(7)}= & \mu \zeta^{(7)}-\frac{1}{2} \mu \zeta^{(3) 2}(\mu-1)[(7 \mu+4)(\rho-\sigma)-3 \sigma] \\
+ & \frac{1}{2} \mu \zeta^{(5)}(\mu-1)(\rho-\sigma)[(7 \mu+1)(\rho-\sigma)-6 \sigma] \\
+ & \frac{1}{8} \mu \zeta^{(3)}(\mu-1)(\rho-\sigma)\left[\left(63 \mu^{3}-33 \mu^{2}-7 \mu+1\right)(\rho-\sigma)^{3}\right. \\
- & \left.\left(96 \mu^{2}-68 \mu-8\right)(\rho-\sigma)^{2} \sigma+(40 \mu-32)(\rho-\sigma) \sigma^{2}-4 \sigma^{3}\right] \\
- & \frac{1}{16} \mu(\mu-1)(\rho-\sigma)^{2}\left[\mu(\rho-\sigma)-\sigma(33]\left[\mu^{4}-47 \mu^{3}+25 \mu^{2}-7 \mu+1\right)(\rho+-\sigma)^{4}\right. \\
& -\left(47 \mu^{3}-80 \mu^{2}+40 \mu-7\right)(\rho-\sigma)^{3} \sigma+\left(25 \mu^{2}-40 \mu+17\right)(\rho-\sigma)^{2} \sigma^{2} \\
& \left.-(7 \mu-7)(\rho-\sigma) \sigma^{3}+\sigma^{4}\right]
\end{aligned}
$$




$$
\begin{aligned}
\zeta^{\prime(9)}= & \mu \zeta^{(9)}-\mu \frac{1}{\rho-\sigma} \zeta^{(3) 3}(\mu-1)[(411 \mu+81)(\rho-\sigma)-330 \sigma]-\mu \zeta^{(3)} \zeta^{(5)} \\
& \cdot(\mu-1)[(9 \mu+5)(\rho-\sigma)-4 \sigma]+\frac{1}{2} \mu \zeta^{(7)}(\mu-1)(\rho-\sigma)[(9 \mu+1)(\rho-\sigma) \\
& -8 \sigma]-\frac{1}{8} \mu \zeta^{(3) 2}(\mu-1)\left[\left(198 \mu^{3}-42 \mu^{2}-80 \mu-3\right)(\rho-\sigma)^{3}\right. \\
& \left.-\left(240 \mu^{2}-116 \mu-75\right)(\rho-\sigma)^{2} \sigma+(70 \mu-51)(\rho-\sigma) \sigma^{2}+41 \sigma^{3}\right] \\
& +\frac{1}{8} \mu \zeta^{(5)}(\mu-1)(\rho-\sigma)\left[\left(99 \mu^{3}-61 \mu^{2}-9 \mu+1\right)(\rho-\sigma)^{3}-\left(160 \mu^{2}-124 \mu\right.\right. \\
& -10) \cdot \\
& \left.\cdot(\rho-\sigma)^{2} \sigma+(70 \mu-60)(\rho-\sigma) \sigma^{2}-6 \sigma^{3}\right]+\frac{1}{16} \mu \zeta^{(3)}(\mu-1)(\rho-\sigma) .
\end{aligned}
$$$$
\cdot\left[\left(429 \mu^{5}-531 \mu^{4}+140 \mu^{3}+20 \mu^{2}-9 \mu+1\right)(\rho-\sigma)^{5}\right.
$$$$
-\left(960 \mu^{4}-1,372 \mu^{3}+428 \mu^{2}+38 \mu-10\right)(\rho-\sigma)^{4} \sigma
$$$$
+\left(770 \mu^{3}-1,190 \mu^{2}+1,320 \mu+20\right)(\rho-\sigma)^{3} \sigma^{2}-\left(280 \mu^{2}-394 \mu+130\right) \text {. }
$$$$
\left.\cdot(\rho-\sigma)^{2} \sigma^{3}+(50 \mu-40)(\rho-\sigma) \sigma^{4}-4 \sigma^{5}\right]-\frac{1}{128} \mu(\mu-1)(\rho-\sigma)^{2} \text {. }
$$$$
\cdot[\mu(\rho-\sigma)-\sigma]\left[\left(715 \mu^{6}-1,525 \mu^{5}+1,335 \mu^{4}-665 \mu^{3}+215 \mu^{2}-35 \mu+5\right)\right. \text {. }
$$$$
\text { . }(\rho-\sigma)^{6}-\left(1,525 \mu^{5}-3,671 \mu^{4}+2,709 \mu^{3}-1,489 \mu^{2}+371 \mu-35\right)(\rho-\sigma)^{5} \sigma
$$$$
+\left(1,265 \mu^{4}-3,189 \mu^{3}+2,873 \mu^{2}-1,099 \mu+165\right)(\rho-\sigma)^{4} \sigma^{2}
$$$$
\left.-575 \mu^{3}-1,369 \mu^{2}+1,049 \mu-275\right)(\rho-\sigma)^{3} \sigma^{3}+\left(215 \mu^{2}-371 \mu+165\right) \text {. }
$$$$
\left.\cdot(\rho-\sigma)^{2} \sigma^{4}-(35 \mu-35)(\rho-\sigma) \sigma^{5}+5 \sigma^{6}\right]
$$

It may, at times, be preferable to multiply these equations by $n^{\prime}$ and write them in the following reduced form

$$
\begin{aligned}
n^{\prime} \sigma^{\prime} & =n \sigma-\left(n-n^{\prime}\right) \rho \\
n^{\prime} \zeta^{\prime(3)} & =n \zeta^{(3)}-\frac{1}{2} n(\mu-1)(\rho-\sigma)^{2}[\mu(\rho-\sigma)-\sigma] \\
n^{\prime} \zeta^{\prime(5)}=n \zeta^{(5)}+\frac{1}{2} n \zeta^{(3)}(\mu-1)(\rho-\sigma)[(5 \mu+1)(\rho-\sigma)-4 \sigma] & -\frac{1}{8} n(\mu-1)(\rho-\sigma)^{2}[\mu(\rho-\sigma)-\sigma]\left[\left(7 \mu^{2}-5 \mu\right.\right. \\
& \left.+1)(\rho-\sigma)^{2}-(5 \mu-5)(\rho-\sigma) \sigma+\sigma^{2}\right]
\end{aligned}
$$

and similarly for higher orders. In computations by means of these equations it is not necessary to find the $\zeta$ coefficients explicitly, but only the "reduced coefficients," $n \zeta^{(3)}, n \zeta^{(5)}$, etc.

\section{DISCUSSION}

It is evident that these formulas are considerably more complex in form than those which refer to the center of curvature. They are included here as supplementary formulas to be applied where the other system is unsuitable. The eleventh order has not been included partly because of its unwieldiness and partly because in the cases where these equations will be most frequently applied, namely, surfaces of small or zero curvature or where the object is near the center of curvature, the inherent aberration will be small and fewer orders will, in general, be required than for the other surfaces of the system. 
The zero points common to all orders of inherent aberration are given by

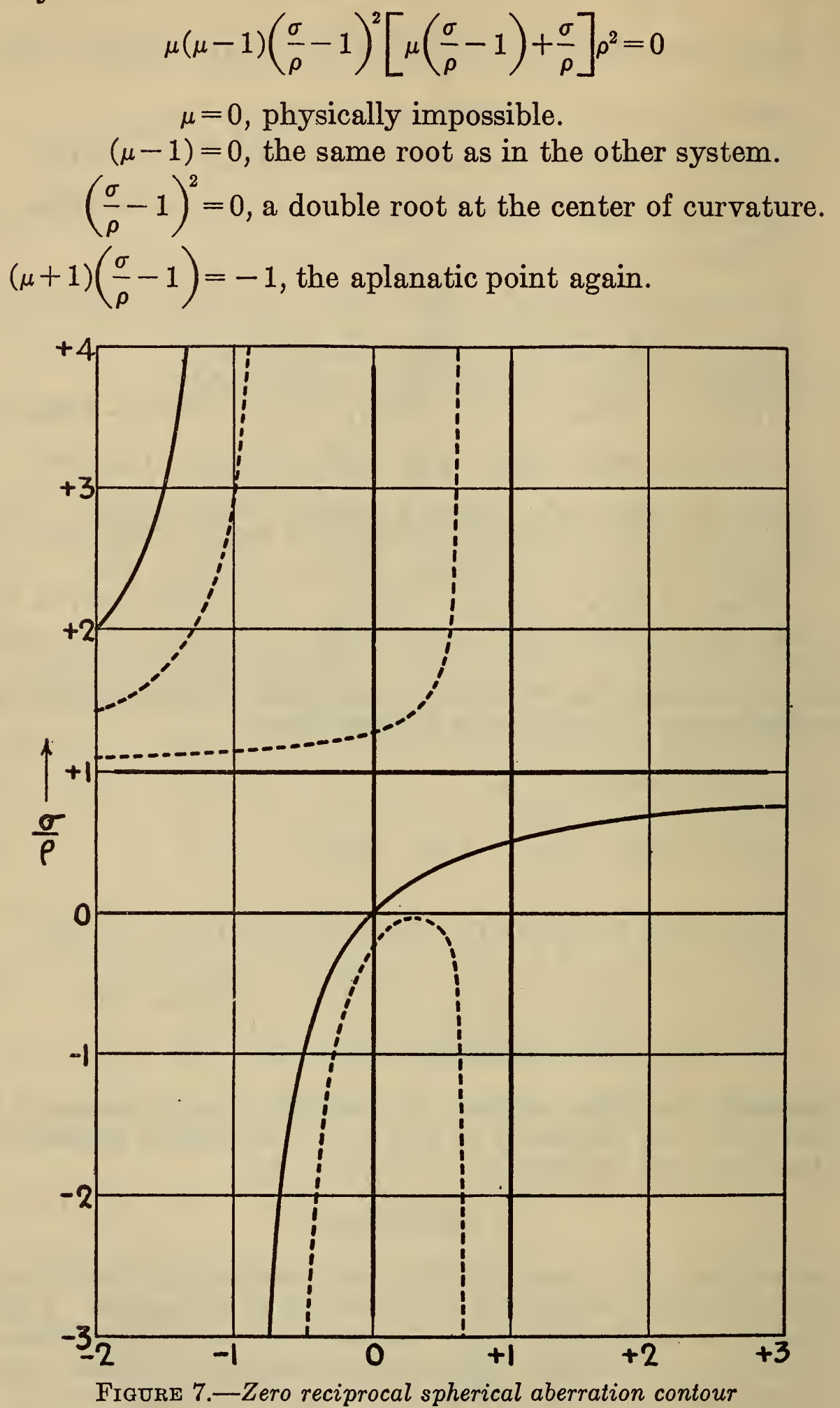

The solid lines indicate zero values of all orders. The dotted curve indicates an additional root of the fifth order. Additional roots of higher orders are not shown.

These roots are shown for $\rho=1$ in Figure 7, as is also an additional root of the fifth order. The additional roots of the higher orders have not been investigated. 
Figures 8 and 9 , corresponding to Figures 4 and 5, show the values of the inherent aberration coefficients for $\mu=1 / 1.5$ and $\mu=1.5$ when $\rho=1$. It is to be remembered that $\mu=1 / 1.5$ corresponds to $\mu^{\prime}=1.5$ in Figure 4.

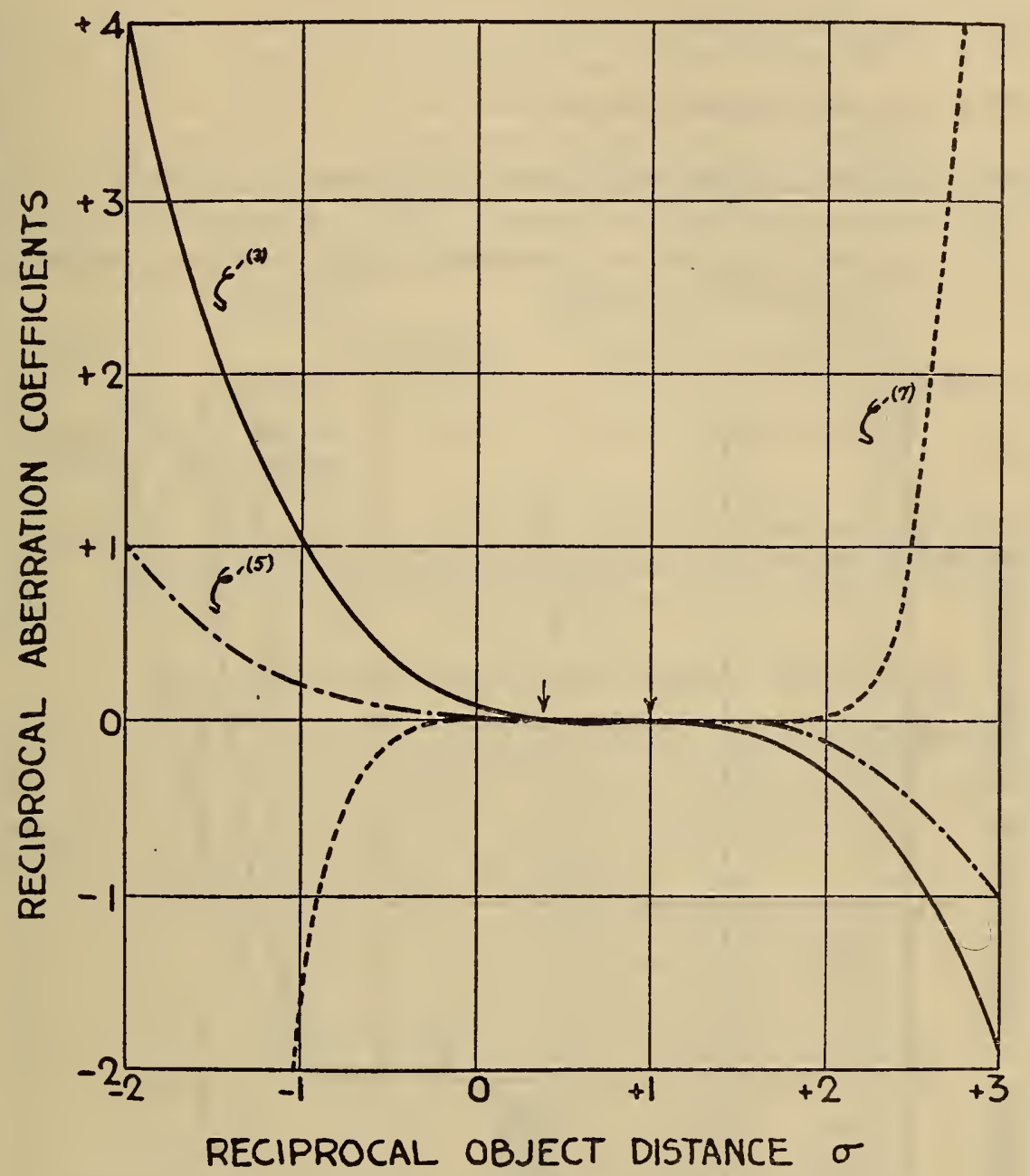

FigdRe 8.-Reciprocal aberration coefficierts for single surface of unit radius, $\mu=1 / 1.5$

\section{INVERSION TO LONGITUDINAL SPHERICAL ABERRATION}

Given the reciprocal aberration of a pencil of rays, the longitudinal aberration may be derived by expanding the brackets in the following equation

$$
\begin{aligned}
S & =\frac{1}{\Sigma}=\frac{1}{\sigma+\zeta^{(3)} h^{2} v+\zeta^{(5)} h^{4} v+\ldots} \\
& \left.=\frac{1}{\sigma\left(1+\frac{1}{\sigma} \zeta^{(3)} h^{2}{ }_{0}+\frac{1}{\sigma} \zeta^{(5)} h^{4} v+\ldots\right.}\right) \\
& =s\left[1+\left(s \zeta^{(3)} h^{2},+s \zeta^{(5)} h^{4}+s \zeta^{(7)} h^{6}, s \zeta^{(9)} h_{0}^{8}+s \zeta^{(11)} h^{10}{ }_{0}\right)\right]^{-1}
\end{aligned}
$$

according to the formula $(1+x)^{-1}=1-x+x^{2}-x^{3}+\ldots$ If the longitudinal aberration be expressed as before. (See Introduction.)

$$
S=s+A h^{2}+B h^{4}+C h^{6}+D h^{8}+E h^{10}
$$


the following formulas are found for converting reciprocal into longitudinal aberration:

$$
\begin{aligned}
& A=-s^{2} \zeta^{(3)} \\
& B=-s^{2}\left(\zeta^{(5)}-s \zeta^{(3) 2}\right) \\
& C=-s^{2}\left(\zeta^{(7)}-2 s \zeta^{(3)} \zeta^{(5)}+s^{2} \zeta^{(3) 3}\right) \\
& D=-s^{2}\left[\zeta^{(9)}-s\left(2 \zeta^{(3)} \zeta^{(7)}+\zeta^{(5) 2}\right)+3 s^{2} \zeta^{(3) 2} \zeta^{(5)}-s^{3} \zeta^{(3) 4}\right] \\
& E=-s^{2}\left[\zeta^{(11)}-2 s\left(\zeta^{(3)} \zeta^{(9)}+\zeta^{(5)} \zeta^{(7)}\right)+3 s^{2}\left(\zeta^{(3) 2} \zeta^{(7)}+\zeta^{(3)} \zeta^{(5) 2}\right)\right. \\
& \left.\quad-4 s^{3} \zeta^{(3) 3} \zeta^{(5)}+s^{4} \zeta^{(3) 4}\right]
\end{aligned}
$$

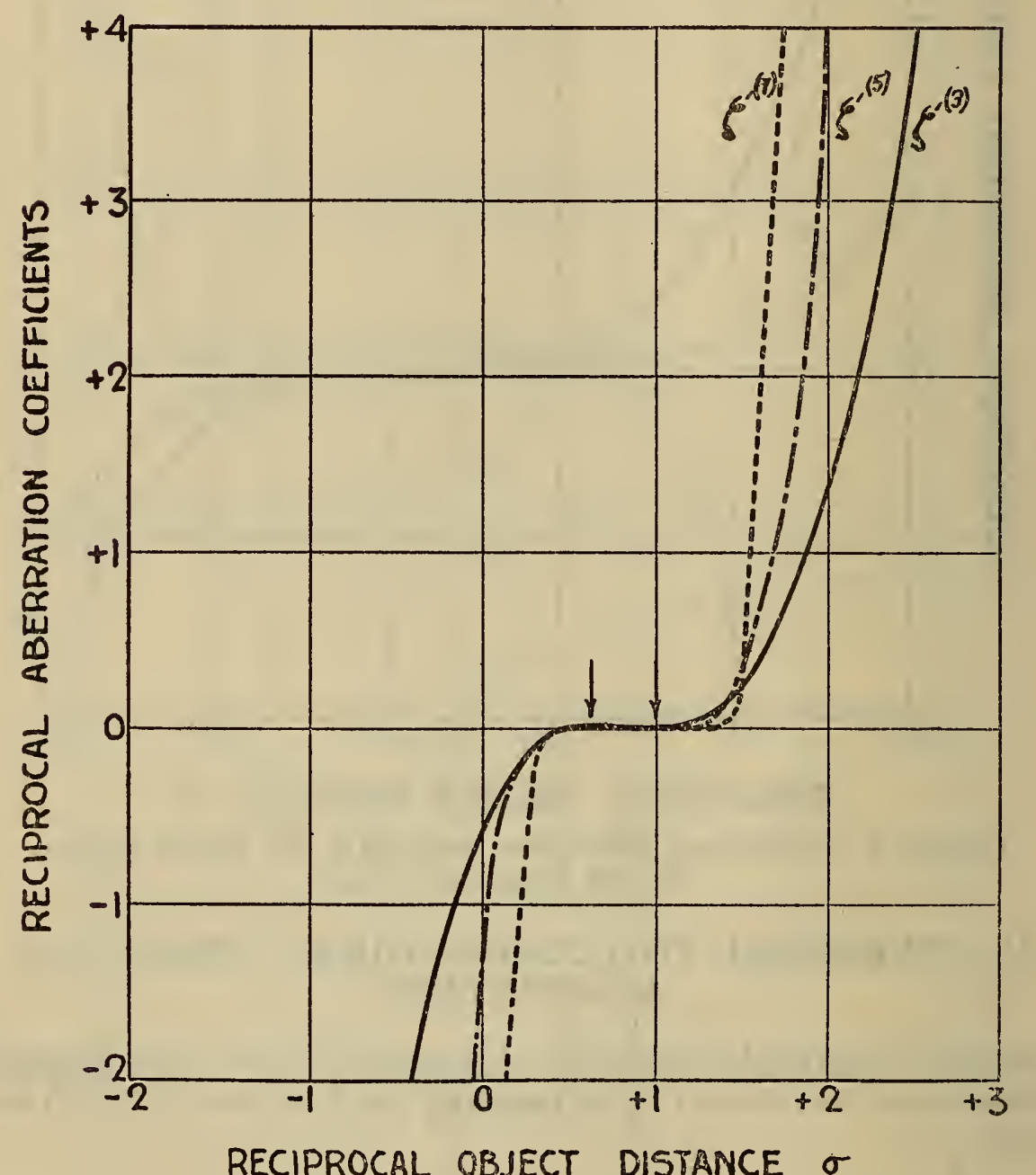

FIGURE 9.-Reciprocal. aberration coefficients for single surface of unit radius, $\mu=1.5$

These formulas are quite general. If the aberration refers to some point other than the vertex of a surface, it is necessary only to replace these symbols by the corresponding ones in the new series. The parameter may be taken as any function of the aperture and of any constants (as, for instance, the constants of a surface of the lens) so long as a convergent series result both for the reciprocal and for the longitudinal aberrations. 


\section{NUMERICAL EXAMPLE}

\section{DETAILS OF CONSTRUCTION OF THE LENS}

As an illustration of the application of the formulas derived in the preceding parts of the paper, the reciprocal spherical aberration of the third, fifth, and seventh orders has been computed for an ordinary landscape lens such as is found in most low-priced cameras. The details of construction of the lens as given by Conrady ${ }^{17}$ and the necessary reciprocals are as follows, except that the decimal point has been changed in order to bring it near the first significant figure both in the lengths and in their reciprocals:

$$
\begin{aligned}
& r_{1}=-0.5556 \\
& n_{1,2}=1.63487 \quad d_{1,2}=0.01 \\
& r_{2}=+1.492 \\
& n_{2,3}=1.54712 \quad d_{2,3}=0.03 \\
& r_{3}=-0.2506 \\
& \begin{aligned}
\rho_{1} & =-1.7999 \\
n^{-1}{ }_{1,2}=0.61167 & \rho_{2}=+0.67024
\end{aligned} \\
& n^{-1}{ }_{2,3}=0.64636 \\
& \rho_{3}=-3.9904
\end{aligned}
$$

The formulas (12), (11), and (28) will be used at the respective surfaces. The final longitudinal aberration will then be derived and compared with the results of trigonometric ray tracing.

\section{FIRST SURFACE; INFINITE OBJECT DISTANCE}

Since $\tau_{1}=0$ and $n_{1}=1$, the simplified formulas (12) may be used at the first surface. The numerical work is conveniently arranged in four columns, one for the paraxial values and one each for the three orders of aberration computed, as follows:

\begin{tabular}{|c|r|c|c|c|}
\hline & Paraxial & Third order & Fifth order & $\begin{array}{c}\text { Seventh } \\
\text { order }\end{array}$ \\
\hline$\frac{1}{n^{\prime} j}$ & 0.61167 & 0.61167 & 0.22885 & 0.08562 \\
$\left(1-\frac{1}{n^{\prime j}}\right)$ & .38833 & .38833 & .77115 & .91438 \\
$\rho_{i}$ & -1.7999 & -1.7999 & -5.8310 & -18.890 \\
$\frac{\rho}{n^{\prime}}$ & -1.9816 & 1.9816 & 1.9816 \\
\hdashline$\frac{\tau}{n^{\prime}} ; \frac{\beta^{\prime}(i)}{n^{\prime i}}$ & -0.69896 & -0.69253 & -1.11381 & -2.1392 \\
\hline
\end{tabular}

where $j=1,1,3$, and 5 in the respective columns.

The first line in this computation need not be written down if the computer so prefers. The next four lines contain the factors of the coefficients of the respective orders. The reciprocal of the intersection distance measured from the center of curvature could now be found for a ray at any height $h^{\prime}{ }_{c 1}$ from the center of curvature after refraction at this surface, this quantity being given by the series

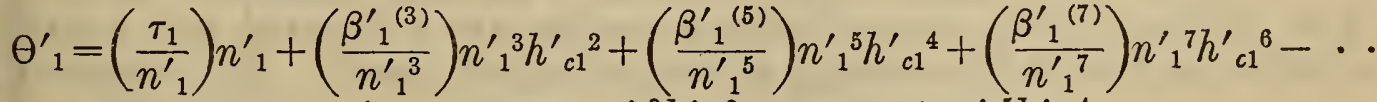

$$
\begin{aligned}
& =-0.69896 n^{\prime}{ }_{1}-0.69253 n^{\prime}{ }_{1}{ }^{3} h^{\prime}{ }_{c 1}{ }^{2}-1.11381 n^{\prime}{ }_{1}{ }^{5} h^{\prime}{ }_{c 1}{ }^{4} \\
& -2.1392 n^{\prime}{ }_{1}{ }^{\prime} h^{\prime}{ }_{c 1}{ }^{6}-\ldots .
\end{aligned}
$$




\section{TRANSFER FROM CENTER OF CURVATURE TO CENTER OF CURVATURE; FIRST SURFACE TO SECOND}

Formulas (19), Section III, 3, are used in transferring the point of reference to the center of curvature of the second surface. The numerical work is most conveniently carried out in two parts, first computing the various terms and factors in the formulas, and second combining them to find the new coefficients, as follows:

\begin{tabular}{|c|c|c|c|c|c|c|c|}
\hline $\begin{array}{l}1 \\
2 \\
3 \\
4 \\
5\end{array}$ & $\begin{array}{r}d \\
n^{\prime} d \\
\kappa n^{\prime} d\end{array}$ & $\begin{array}{l}+2.0576 \\
+3.3639 \\
+1.00379 \\
+1.00759\end{array}$ & $\begin{array}{l}\kappa \\
\kappa^{2} \\
\kappa^{4} \\
\kappa^{6} \\
\kappa^{8}\end{array}$ & \multicolumn{2}{|c|}{$\begin{array}{l}+0.29840 \\
\quad .089043 \\
.79287 \cdot 10^{-2} \\
.70600 \cdot 10^{-3} \\
.62864 \cdot 10^{-4}\end{array}$} & $\begin{array}{l}\frac{\beta^{\prime(3) 2}}{n^{\prime 8}} \\
\frac{\beta^{\prime(3)} \beta^{\prime(3)}}{n^{\prime 8}} \\
\frac{\beta^{\prime(8) 3}}{n^{\prime 6}}\end{array}$ & $\begin{array}{r}+0.47960 \\
+.77135 \\
-.33214\end{array}$ \\
\hline \multirow{4}{*}{$\begin{array}{l}6 \\
7 \\
8\end{array}$} & \multirow[b]{2}{*}{$\begin{array}{l}\frac{\tau^{\prime}{ }^{\prime}}{n^{\prime}} ; \frac{\beta_{1}^{\prime}{ }_{1}^{(i)}}{n_{1}^{\prime}} \\
\text { k-term } \\
\kappa^{2} \text {-term } \\
\\
\text { Sum }\end{array}$} & Paraxial & \multicolumn{2}{|c|}{ Third order } & \multicolumn{2}{|c|}{ Fifth order } & Seventh order \\
\hline & & $\begin{array}{c}-0.69896 \\
\end{array}$ & \multicolumn{2}{|c|}{-0.69253} & \multicolumn{2}{|c|}{$\begin{array}{l}-1.11381 \\
+1.44425\end{array}$} & -2.1392 \\
\hline & \multirow{2}{*}{$\begin{array}{l}\text { Sum } \\
\frac{\tau_{2}}{n_{2}} ; \frac{\beta_{2}(i)}{n_{2}^{i}}-\ldots\end{array}$} & . & . & -........ & \multicolumn{2}{|c|}{$\begin{array}{r}+.33044 \\
\end{array}$} & +.0391 \\
\hline & & -.20857 & -.5490 & $9 \cdot 10^{-2}$ & +.233 & $29 \cdot 10^{-3}$ & $+.0246 \cdot 10^{-4}$ \\
\hline
\end{tabular}

In this computation the value of $d$ is found from the original data $\left(d_{c 1 c 2}=d_{v 1 v_{2}}-r_{1}+r_{2}\right)$, then $\kappa$ and its powers and multiples are computed, $n^{\prime} d$ being found incidentally to the computation of $\kappa$. Line 6 is copied from the last line of the computation in the preceding section. The "sums" in line 9 are the numerical values of the quantities in brackets in the formulas, which, when multiplied by the proper power of $\kappa$ give the coefficients for the object pencil at the second
surface.

\section{SECOND SURFACE AND TRANSFER TO THIRD; CENTER OF CURVATURE TO VERTEX}

The aberration at the second surface is computed by means of formulas (11). As may be seen below, the computation falls readily into three parts. In lines 1 to 10 the various monomial and binomial quantities and their powers which appear in the formulas are computed. In lines 11 to 14 are computed the four bracketed quantities which appear in the $\frac{\beta^{\prime(3)}}{n^{\prime 3}}-$ inherent, the $\frac{\beta^{\prime(5)}}{n^{\prime 5}}-$ inherent, the $\frac{\beta^{\prime(7)}}{n^{\prime 7}}\left(\frac{\beta^{(3)}}{n^{3}}\right)$, and the $\frac{\beta^{\prime(7)}}{n^{\prime 7}}$-inherent terms respectively. ${ }^{18}$ In each case the three products composing the quantity are written in the first three columns and their sum is written in the last column. In lines 15 to 20 the various terms in the formulas are evaluated and added together to find the constants of the refracted pencil. The inherent aberration appears in line 16 , the terms containing the factor $\frac{\beta^{(3)}}{n^{3}}$ are in line 17 , and so on. 
In the transfer to the vertex of the third surface, the first part of the computation, lines 25 to 29 , has been written below the second part, lines 20 to 24 in order to eliminate the recopying of line 20 . Moreover, the computation differs slightly from that in the preceding section in the use of formulas (20) instead of (19).

The numerical work, then, is as follows:

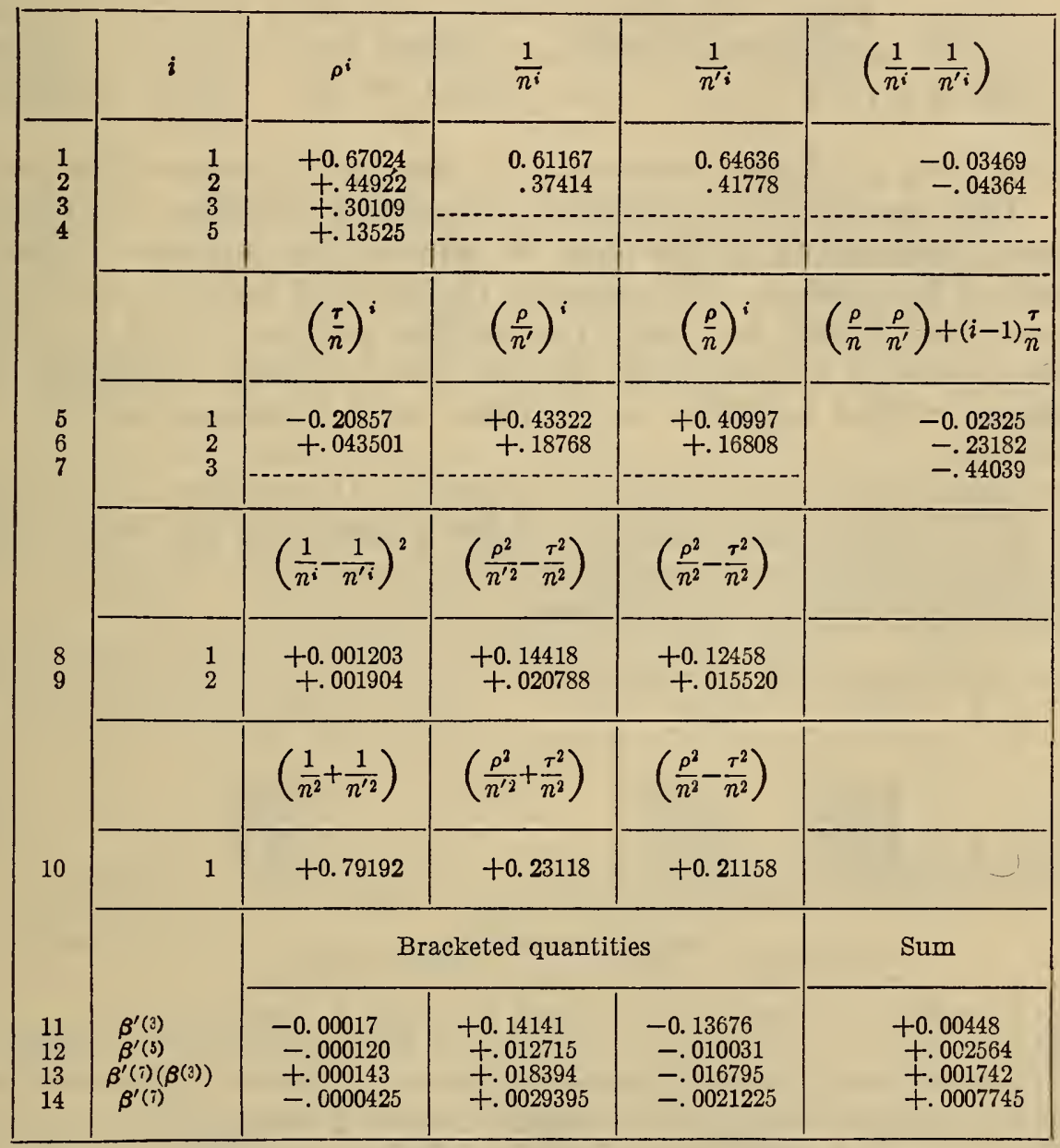

\begin{tabular}{|c|c|c|c|c|c|c|c|}
\hline & & Paraxial & \multicolumn{2}{|c|}{ Third order } & \multicolumn{2}{|c|}{ Fifth order } & Seventh order \\
\hline 15 & $\frac{\tau_{2}}{n_{2}} ; \frac{\beta_{2}(i)}{n_{2} i}$ & -0.20857 & \multicolumn{2}{|c|}{$-0.54909 \cdot 10^{-2}$} & \multicolumn{2}{|c|}{$+0.23329 \cdot 10^{-3}$} & $+0.0246 \cdot 10^{-4}$ \\
\hline $\begin{array}{l}16 \\
17 \\
18 \\
19\end{array}$ & $\begin{array}{l}\text { Inher. } \\
(\beta(3)) \\
(\beta(5)) \\
(\beta(3) 2)\end{array}$ & -.02325 & \multicolumn{2}{|c|}{ (n) } & $\begin{array}{r}-.21 \\
+.02 \\
\end{array}$ & $\begin{array}{l}18 \cdot 10^{-3} \\
31 \cdot 10^{-3} \\
-1 .-1-1 .-\end{array}$ & $\begin{array}{r}-.3244 \cdot 10^{-4} \\
+.0321 \cdot 10^{-4} \\
-.0119 \cdot 10^{-4} \\
+.0035 \cdot 10^{-4}\end{array}$ \\
\hline 20 & $\frac{\tau_{2}^{\prime}}{n^{\prime}} ; \frac{\beta_{2}^{\prime}{ }_{2}^{(i)}}{n^{\prime} 2^{i}}$ & -.23182 & \multicolumn{2}{|c|}{$-.6992 \cdot 10^{-2}$} & \multicolumn{2}{|c|}{$+.0466 \cdot 10^{-3}$} & $-.2761 \cdot 10^{-4}$ \\
\hline $\begin{array}{l}21 \\
22\end{array}$ & $\begin{array}{l}(\kappa) \\
\left(k^{2}\right)\end{array}$ & - & (n) & . & \multicolumn{2}{|c|}{$-.6975 \cdot 10^{-3}$} & $\begin{array}{l}+.1239 \cdot 10^{-4} \\
-.9275 \cdot 10^{-4} \\
\end{array}$ \\
\hline 23 & Sum & 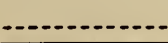 & -..-- & $\ldots$ & \multicolumn{2}{|c|}{$-.6509 \cdot 10^{-3}$} & $-1.0797 \cdot 10^{-4}$ \\
\hline 24 & $n \sigma_{3} ; n \zeta$ & -1.16660 & \multicolumn{2}{|c|}{-.7827} & \multicolumn{2}{|c|}{-.7709} & -1.3529 \\
\hline 25 & $d$ & -1.462 & $n_{\kappa}$ & \multicolumn{2}{|c|}{+3.2527} & $\frac{\beta(8) 2}{n^{6}}$ & $+0.48888 .10^{-1}$ \\
\hline 26 & $1 / n^{\prime}$ & .64636 & $(n \kappa)^{2}$ & \multicolumn{2}{|c|}{10.5801} & & \multirow{3}{*}{$-.03258 \cdot 10^{-5}$} \\
\hline 27 & $n^{\prime} \times d$ & -4.7554 & $\left(n_{\kappa}\right)^{4}$ & \multicolumn{2}{|c|}{ 1. $11939 \cdot 10^{2}$} & $\frac{\beta(0) p(0)}{n^{8}}$ & \\
\hline 28 & $\left(n^{\prime} k d\right)^{2}$ & +22.614 & $(n x)^{6}$ & \multirow{2}{*}{\multicolumn{2}{|c|}{$\begin{array}{l}1.18433 \cdot 10^{3} \\
1.25303 \cdot 10^{4}\end{array}$}} & & \\
\hline 29 & $\sigma$ & -.75404 & $\left(n_{k}\right)^{8}$ & & & $\frac{p}{n^{2}}$ & $-.3418 \cdot 10^{-8}$ \\
\hline
\end{tabular}




\section{THIRD SURFACE AND INVERSION TO LONGITUDINAL ABERRATION}

The aberration due to the third surface of the lens might also be computed by the same method as was used at the second surface. However, it is desired to illustrate the use of formulas (28), and moreover it is preferable to have the final aberration referred to the back vertex without the additional transfer from the center of curvature to the vertex. Since the image is in air, then $n^{\prime}$ is unity, $\mu=n$, equations (28) are identical with equations (27), and the "reduced" coefficients, $n^{\prime} \sigma^{\prime}, n^{\prime} \zeta^{\prime(3)}, \ldots$, are equal to the actual coefficients, $\sigma^{\prime}, \zeta^{\prime(3)}, \ldots$

In lines 1 to 4 of the computation the preliminary data are prepared. The bracketed quantities computed in lines 5 to 22 are given the designation of the term in which they appear. The various sums of the powers of $n$ appear in the first column of numbers. These are multiplied by the appropriate powers of $(\rho-\sigma)$ and $\sigma$, and the products are written in the next column. Finally, these products are added together to give the total value appearing in the last column.

The coefficients of longitudinal spherical aberration are computed in lines 28 to 34 by formulas (29), Part V, in much the same manner as a transfer is made to a new point of reference.

These computations are as follows:

\begin{tabular}{|c|c|c|c|c|c|c|c|}
\hline \multirow[b]{2}{*}{$\begin{array}{l}\text { (1) } \\
(2) \\
(3) \\
\text { (4) }\end{array}$} & $i$ & $n^{i}$ & $(\rho-\sigma)^{i}$ & \multicolumn{4}{|c|}{$\sigma^{i}$} \\
\hline & $\begin{array}{l}1 \\
2 \\
3 \\
4\end{array}$ & $\begin{array}{l}1.54712 \\
\text { 2. } 39358 \\
\text { 3. } 70316 \\
5.7292\end{array}$ & $\begin{array}{r}-3.2364 \\
+10.4743 \\
-33.899 \\
+109.711\end{array}$ & \multicolumn{4}{|c|}{$\begin{array}{r}-0.75404 \\
+.56858 \\
-.42873 \\
+.32328\end{array}$} \\
\hline & \multicolumn{6}{|c|}{ Bracketed quantities } & Sum \\
\hline $\begin{array}{l}(5) \\
(6)\end{array}$ & $\zeta^{\prime(5)}$ & & $(5 n+1)$ & 8.7356 & $\begin{array}{l}\cdot(\rho-\sigma) \\
-4 \sigma\end{array}$ & $\begin{array}{r}-28.272 \\
+3.016\end{array}$ & -25.256 \\
\hline $\begin{array}{l}\text { (7) } \\
(8) \\
(9)\end{array}$ & $\zeta^{\prime(5)}($ & {$[n h)$.} & $\stackrel{\left(7 n^{2}-\right.}{-(5 n-5)}$ & $\begin{array}{r}10.0195 \\
-2.7356\end{array}$ & $\begin{array}{l}\cdot(\rho-\sigma)^{2} \\
\cdot(\rho) \sigma \\
+\sigma^{2}\end{array}$ & $\begin{array}{r}+104.947 \\
-6.6759 \\
+.5686\end{array}$ & +98.840 \\
\hline $\begin{array}{l}\text { (10) } \\
\text { (11) }\end{array}$ & $\xi^{\prime(i)}(\zeta$ & & $(7 n+4)$ & 14.8298 & $\frac{\cdot(\rho-\sigma)}{-3 \sigma}$ & $\begin{array}{r}-47.995 \\
+2.2621\end{array}$ & -45.733 \\
\hline $\begin{array}{l}(12) \\
(13)\end{array}$ & $\zeta^{\prime(i)}$ & $\left(\xi^{(5)}\right)$ & $(7 n+1)$ & 11.8298 & $\begin{array}{l}\cdot(\rho-\sigma) \\
-6 \sigma\end{array}$ & $\begin{array}{r}-38.236 \\
+4.524\end{array}$ & -33.762 \\
\hline $\begin{array}{l}(14) \\
(15) \\
(16) \\
\text { (17) }\end{array}$ & $\zeta^{\prime}(7)$ & & $\begin{array}{c}\left(63 n^{3}-.\right) \\
-\left(96 n^{2}-.\right) \\
(40 n-32)\end{array}$ & $\begin{array}{r}144.481 \\
-116.579 \\
29.8848\end{array}$ & $\begin{array}{l}\cdot(\rho-\sigma)^{3} \\
\cdot(\rho)^{2} \sigma \\
\cdot(\rho)^{2} \\
-4 \sigma^{3}\end{array}$ & $\begin{array}{r}-4,897.8 \\
+920.75 \\
-54.99 \\
+1.71\end{array}$ & $-4,030.3$ \\
\hline
\end{tabular}




\begin{tabular}{|c|c|c|c|c|c|c|c|}
\hline \multirow[t]{2}{*}{$\begin{array}{l}(18) \\
(19) \\
(20) \\
(21) \\
(22)\end{array}$} & $\zeta^{\prime(7)}(\operatorname{Inh.})$ & $\begin{array}{r}\left(33 n^{4}-. .\right) \\
-\left(47 n^{3}-. .\right) \\
\left(25 n^{2}-.\right) \\
-(7 n-7)\end{array}$ & $\begin{array}{r}65.0247 \\
-37.446 \\
14.9547 \\
-3.8298\end{array}$ & $\begin{array}{l}\cdot(\rho-\sigma) \\
\cdot(\rho)^{3} \sigma \\
\cdot(\rho)^{2} \sigma^{2} \\
\cdot(\rho) \sigma^{3} \\
+\rho^{4}\end{array}$ & \multicolumn{2}{|c|}{$\begin{array}{r}+7,133.92 \\
-957.16 \\
+89.06 \\
-5.31 \\
+.32\end{array}$} & $+6,260.8$ \\
\hline & & Paraxial & \multicolumn{2}{|c|}{ Third order } & Fifth order & & eventh order \\
\hline $\begin{array}{l}(23) \\
(24) \\
(25) \\
(26) \\
(27)\end{array}$ & $\begin{array}{c}n \sigma_{3} ; n \zeta_{3}(i) \\
\text { Inher. } \\
\left(\zeta^{(3)}\right) \\
\left(\zeta^{(5)}\right) \\
\left(\zeta^{(3) 2)}\right.\end{array}$ & $\begin{array}{l}-1.16660 \\
+2.1832\end{array}$ & \multicolumn{2}{|c|}{$\begin{array}{l}-0.7827 \\
+18.854 \\
\end{array}$} & $\begin{aligned} & -0.7709 \\
+ & 465.88 \\
- & 27.08\end{aligned}$ & \multicolumn{2}{|c|}{$\begin{array}{c}-1.3529 \\
+14,755 . \\
-1,080.2 \\
-35.7 \\
+11.9\end{array}$} \\
\hline $\begin{array}{l}(28) \\
(29) \\
(30)\end{array}$ & $\begin{array}{r}\sigma_{3}^{\prime} ; \zeta_{3}^{\prime}{ }_{3}^{(i)} \\
s-\text { term } \\
s^{2} \text {-term }\end{array}$ & +1.0166 & \multicolumn{2}{|c|}{+18.071} & $\begin{array}{l}+438.03 \\
-321.23\end{array}$ & & $\begin{array}{l}13,650 \\
15,573 \\
+5,710\end{array}$ \\
\hline \multirow{2}{*}{$\begin{array}{l}(31) \\
(32)\end{array}$} & \multirow{2}{*}{$s^{\prime}, A^{\prime}, B^{\prime}, C^{\prime}$} & - & \multicolumn{2}{|c|}{ - } & +116.80 & & $+3,787$ \\
\hline & & +.98367 & \multicolumn{2}{|c|}{-17.486} & -113.02 & & $-3,664$ \\
\hline $\begin{array}{l}(33) \\
(34)\end{array}$ & $\begin{array}{c}s^{2} \\
\zeta^{\prime(3) 2}\end{array}$ & \multicolumn{2}{|c|}{$\begin{aligned} & 0.96761 \\
+ & 326.56\end{aligned}$} & \multicolumn{2}{|c|}{$\begin{array}{c}\zeta^{\prime}(3) \zeta^{\prime}(5) \\
\zeta^{\prime}(3) 3\end{array}$} & & $\begin{array}{l}+7,915.6 \\
+5,901.3\end{array}$ \\
\hline
\end{tabular}

It is more or less customary to change the dimensions of a lens proportionately so that the focal length is $1,000 \mathrm{~mm}$ when plotting the aberrations. This is done to a satisfactory approximation in the present case by merely changing the decimal point. The intersection distance measured from the back vertex for an image ray at height $h^{\prime}{ }_{3}$ is then given by the series

$$
\begin{aligned}
S_{3}^{\prime} & =s^{\prime}{ }_{3}+A^{\prime} h^{\prime}{ }_{3}{ }^{2}+B^{\prime} h_{3}^{\prime}{ }_{3}+C^{\prime} h^{\prime}{ }_{3}^{6} \\
& =983.67-0.017486 h^{\prime}{ }_{3}^{2}-0.00011302 h^{\prime}{ }_{3}^{4}-0.000003664 h^{\prime}{ }_{3}^{6}
\end{aligned}
$$

\section{COMPARISON WITH RAY TRACING}

In comparing the results of algebraic and trigonometric computations it is well to keep in mind the following essential difference between the two: In the trigonometric work the longitudinal spherical aberration is found as the difference between the intersection lengths. of a paraxial and another ray. If either length is deternined with. an uncertainty of $0.01 \mathrm{~mm}$, the uncertainty in the aberration thus. determined is at least as large as that. In the algebraic series, on the other hand, the spherical aberration is expressed as one or more correction terms to be applied to the paraxial focal length, and accordingly the aberration may sometimes be found with an uncertainty of $0.001 \mathrm{~mm}$ or less when the intersection lengths are not known to within $0.1 \mathrm{~mm}$.

For the lens in our example Conrady gives the following values obtained by tracing a ray with the use of five place tables:

$$
\begin{array}{ll}
h_{1}=\quad 30.0 & \alpha^{\prime}{ }_{3}=1.8253^{\circ} \\
s^{\prime}{ }_{3}=+983.67 & S^{\prime}{ }_{3}=967.13
\end{array}
$$

Long. Spher. $=-16.54$ 
For purposes of comparison, the algebraic aberration must be determined for the same ray, that is

$$
h^{\prime}{ }_{3}={S^{\prime}}_{3} \sin \alpha^{\prime}{ }_{3}=967.13 \sin 1.8253^{\circ}=30.805
$$

This value substituted into the final series in section 5 , above, gives:

$$
\begin{array}{lr}
A^{\prime} h^{\prime}{ }^{3}=-16.593 & s^{\prime}=+983.67 \\
B^{\prime} h^{\prime}{ }_{3}=-.1018 & \text { Long. Spher. }=-16.698 \\
C^{\prime} h^{\prime}{ }_{3}{ }^{8}=-.0031 & S^{\prime}=+966.972 \\
\text { Total }=-16.698 &
\end{array}
$$

These results are shown in Figure 10.

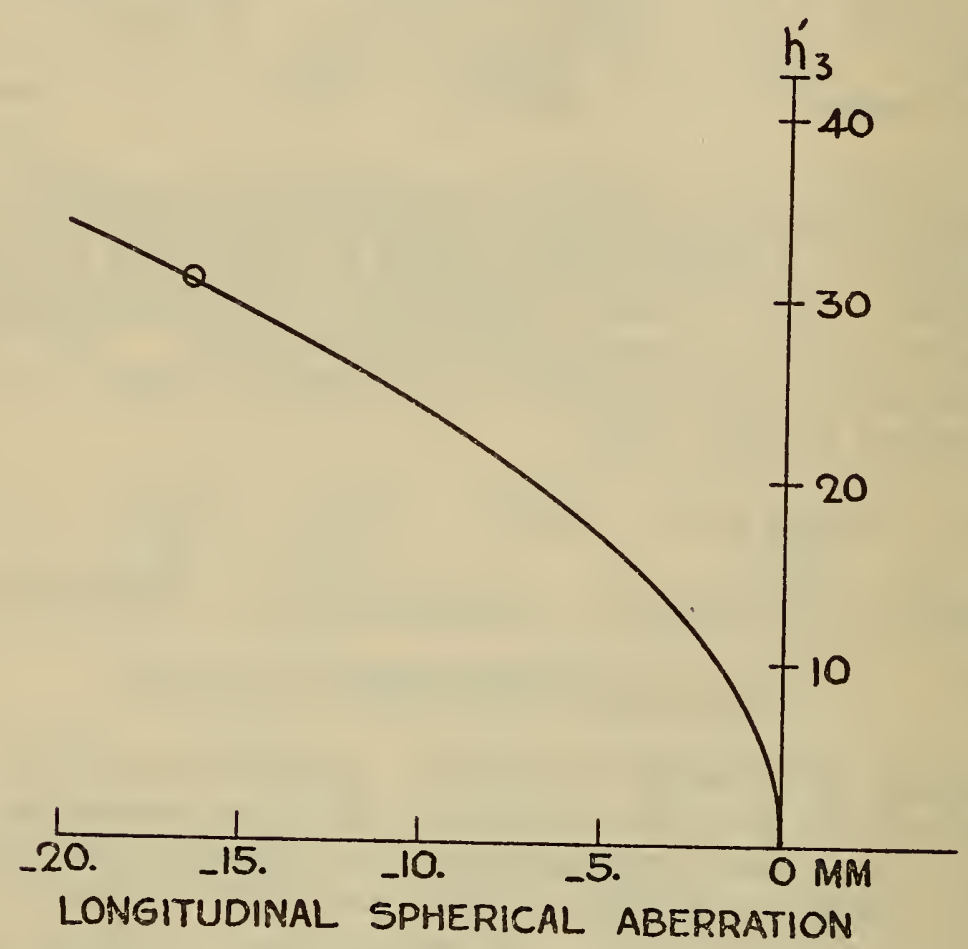

FIGURE 10.-Spherical aberration of "landscape" lens

The curve shows the aberration of this lens as given by the accompanying calculation. The circle indicates a value obtained by trigonometric ray tracing using 5 place tables. The curve as shown corresponds to a back focal length of $983.6 \mathrm{~mm}$. The horizontal scale, however, is twice the vertical.

In order to determine whether the discrepancy of $0.158 \mathrm{~mm}$ between the two values of Long. Spher. is due more largely to the trigonometric or to the algebraic computation, a seven-figure trigonometric computation has been carried out. Its results are as follows:

$$
\begin{aligned}
& s^{\prime}=983.591 \\
& S^{\prime}{ }_{3}=966.888 \quad a^{\prime}{ }_{3}=1.82576^{\circ} \\
& \text { Long. Spher. }=-16.703
\end{aligned}
$$

When this more precise value is taken as a standard of comparison it may be seen that the algebraic computation, even if only the third spherical order term be considered, gives a better value for the spherical aberration than does the five-figure trigonometric computation. Moreover, if the fifth order term is included the resulting aberration differs but little from that given by the seven-place work. The sev- 
enth order term is negligible in the present case, but with larger relative apertures it would become more prominent.

It is interesting to note that Conrady computes the third order spherical aberration to be -16.702 , which is virtually identical with the total aberration given by the 7-place computation, and then states that this value "agrees very well with the more exact trigonometric amount, -16.54 , showing that the higher spherical aberration is small." The difference between the two values of the third order aberration may be considered as due to the causes mentioned in Part I, section 2. On the other hand, the close agreement between the first two paraxial image distances mentioned above appears to be largely fortuitous.

Although a final appraisal of the values of algebraic $v$. trigonometric computations should not be based upon one numerical example alone, still the following estimate, based upon this computation along with others which the author has made, may well be given here. The algebraic method is believed to be capable of giving as dependable results as the trigonometric in all practical cases. As a general rule, an exactness comparable to that given by 5-figure ray tracing may be obtained for apertures up to approximately $f / 10$ by computing the third order only, and for apertures up to approximately $f / 6$ by computing the third and fifth orders. These stated apertures will vary widely, tending to be smaller for meniscus than for symmetrical lenses. The labor involved in computing the paraxial and third order quantities by the method described in this paper is about the same as that of tracing one paraxial and one rim ray, that involved in computing also the fifth order is about the same as that of tracing one paraxial and two other rays. However, the possibility exists of compiling tables of the series aberrations, for instance as functions of the index of refraction and the reciprocal object distance, and thus of reducing the work to a minimum.

\section{DISCUSSION}

\section{CONVERGENCE OF ABERRATION SERIES}

The validity of series expressions of aberration has been questioned on the ground that the magnitude of the terms which are neglected is not known. In fact Baker and Filon ${ }^{19}$ have pointed out two cases in which these series actually become nonconvergent. There are analogous cases in the system of equations developed in the foregoing paragraphs, and these will be briefly discussed here.

In one case of nonconvergence the image distance for rays at some finite distance from the axis is infinite and, accordingly, the longitudinal aberration is infinite and can be expressed by no convergent series. In reciprocal aberration this case can be seen merely to be one of a zero value of the series. Nevertheless, analagous difficulty arises in the reciprocal series when a ray passes through the point of reference so that the reciprocal distance, and hence the reciprocal aberration, becomes infinite. This difficulty, however, can easily be avoided by a proper choice of the point of reference.

The second case mentioned by Baker and Filon concerns aberration series which are written in terms of the inclination of the ray in the

\footnotetext{
19 Baker and Filon, On an Empirical Formula for the Longitudinal Spherical Aberration of a Thick Lens; Trans. Opt. Soc. London, vol. 20, pp. 67-92, 1918-19. See particularly pp. 74-76.
} 
image space and involves longitudinal and reciprocal series alike. It was shown that two rays in the image pencil may have equal inclinations but unequal aberrations so that the aberration is a doublevalued function of the independent variable. Manifestly no series can be convergent for both values.

In the present system of equations there is the following analagous case: Let $S T$ (fig. 11) be the caustic curve of a pencil of rays. If the origin be chosen as at $O$ so that a normal, $N$, to the caustic curve passes through it, then for every ray, $R_{\mathrm{B}} S_{\mathrm{B}}$, tangent to the curve beyond $N$, there is another ray, $R_{\mathrm{A}} S_{\mathrm{A}}$, such that the normals, $O Q$ and $O P$, of the two rays are equal. There are, then two values of the aberration for a single value of the variable, $h$, and consequently no series can be convergent throughout this interval.

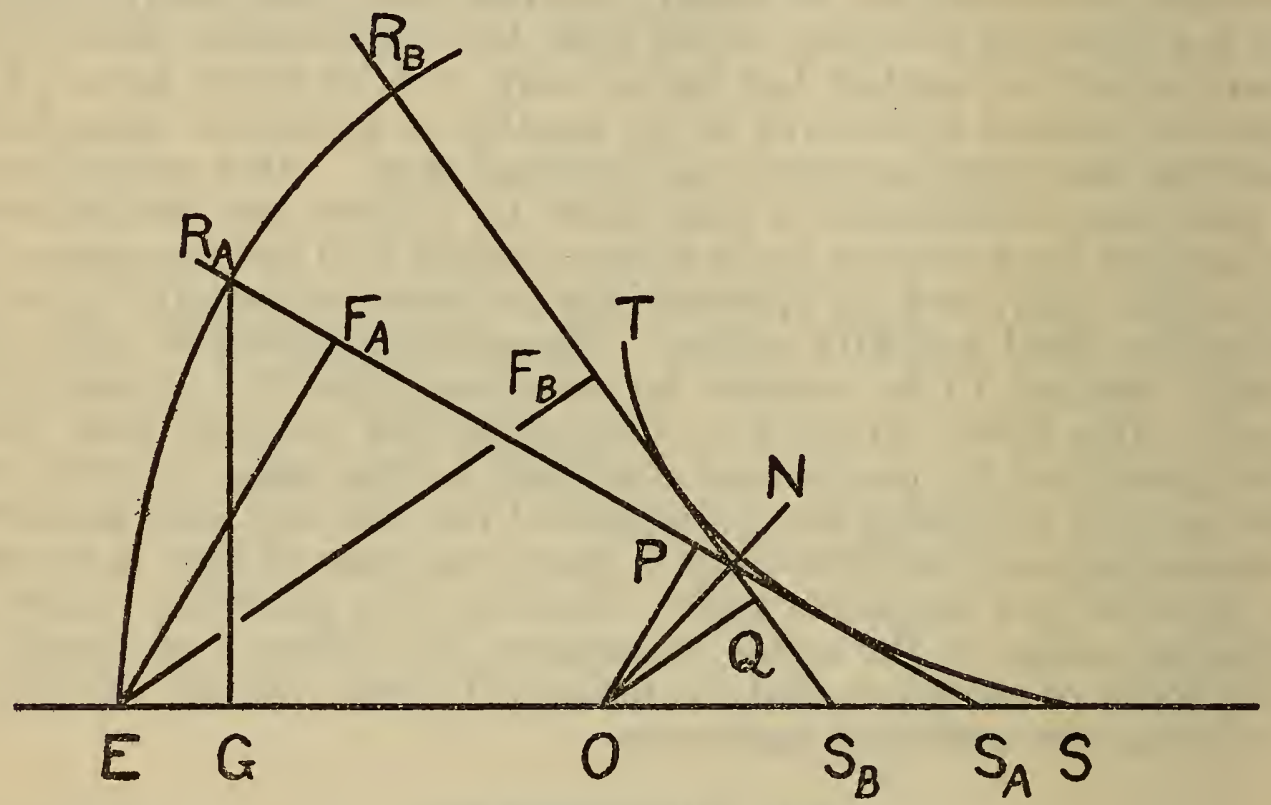

FIGURE 11.-Diagram illustrating nonconvergence of aberration series

However, by choosing a suitable point of reference, this difficulty is avoided, or at least the interval of convergence may be sufficiently extended for practical purposes.

Without stopping for the proof for other types of series, it may be stated as a more or less general rule that, if any aberration series be written using as a variable a length measured from a point on the axis to a point on the ray in some specified manner, than an absolute limit to the interval of convergence of the series is reached when the specified point on the ray coincides with the point of tangency of the ray with the caustic curve. In practice, however, long before this limit is reached the series will become so slowly convergent as to be of no practical value. It is to be observed, however, that nonconvergence can be avoided in the proposed system by a proper choice of the point of reference, while Baker and Filon found it necessary to use a function of the incident ray rather than of the refracted ray as the variable.

This is objectionable on account of the indefiniteness inherent in using a function of the incident ray. For example, if $h$ is given in $S_{4} P_{4}$ (fig. 1), $S^{\prime}{ }_{4}$ may be found from equation (1). However, this determines only one point on the ray since the ray might traverse the lens by a different path and still intersect the axis at the same point. Even if the height of incidence on the last surface is used as the ordi- 
nate and the axial point determined as before, the exact direction of the ray is not known unless the radius of the surface is given also. On the other hand, in the proposed system when the axial point is found by equation (1), the position of the ray is competely determined by a simple geometrical construction. Moreover, the ratio (or in the case of reciprocal aberration, the product) of these two quantities equals the sine of the angle of inclination of the ray while in the two systems just mentioned there is no direct relation.

\section{RELATION TO PATH DIFFERENCE EQUATIONS}

If it is desired to find the path difference between the two rays at some point on the axis, in order to work out the diffraction effects according to formulas such as those published by L. C. Martin, ${ }^{20}$ a further advantage is gained in measuring the aperture along a line perpendicular to the ray rather than to the axis. In Martin's formula the independent variable is the height of intersection of the ray with a wave front through a fixed axial point. If $E R_{A} R_{B}$ (fig. 11) is a wave front, then $R_{A} G$ is such a height. Plainly an axial aberration series could not easily be written using this variable since the shape of the wave front is not usually known. If the wave front were exactly spherical then it is evident that $R_{A} G$ would be exactly equal to $E F$ which is the variable used in the present paper. Now, since wave fronts do not ordinarily depart from sphericity by more than one or two wave lengths, or $0.001 \mathrm{~mm}$, when $h$ equals, say, $20 \mathrm{~mm}$, the two lengths could be considered equal in most cases without appreciable error, so that the coefficients of the series derived in this paper could be substituted directly into Martin's equations.

A further investigation of the relation between reciprocal aberration and path length differences has been begun, and exact equations have been derived. It is expected that these will be published soon.

\section{CONCLUSION}

Besides the theoretical interest which attaches to the equations derived in this paper, there also is believed to be a considerable possibility of their practical application. No optical computer to-day questions the value of the third order aberrations developed by Seidel, and yet Berek ${ }^{21}$ states that "L. Seidel himself did not consider the aberration formulas of the third order which he published between 1853-1856 suitable for practical purposes and, therefore, furnished trigonometrical tracing formulas to the C. A. Steinheil Optical Co. (1866)." Further, it may be stated that the mathematical tools furnished by Seidel lay idle for about 60 years, and only recently their usefulness, in spite of their restrictions, has been recognized. Although the fifth order, up to the present time, has been considered too unwieldy to be useful, still it is not impossible that it, like the third order, will in time be brought into a simpler form adaptable to numerical work. In addition to this there is the possibility of the compilation of tables of these aberrations which would shorten considerably the work of computing.

Washington, June 16, 1932.

${ }^{20}$ L. C. Martin, A Physical Study of Spherical Aberration, Trans. Opt. Soc., London, vol. 23, pp. 63-92 1921-22.

21 Berek, p. 41, see footnote 11, p. 192.

$127984-32-8$ 